\title{
NCOA4 maintains murine erythropoiesis via cell autonomous and non-autonomous mechanisms
}

\author{
Naiara Santana-Codina, ${ }^{1, \star}$ Sebastian Gableske, ${ }^{1, \star}$ Maria Quiles del Rey, ${ }^{1}$ \\ Beata Małachowska, ${ }^{2,3}$ Mark P. Jedrychowski, ${ }^{1,4}$ Douglas E. Biancur, ${ }^{1}$ \\ Paul J. Schmidt, ${ }^{5}$ Mark D. Fleming, ${ }^{5}$ Wojciech Fendler, ${ }^{1,2}$ J. Wade Harper, ${ }^{4, \#}$ \\ Alec C. Kimmelman ${ }^{6, \#}$ and Joseph D. Mancias ${ }^{1}$
}

Haematologica 2019

Volume 104(7):1342-1354

${ }^{1}$ Division of Genomic Stability and DNA Repair, Department of Radiation Oncology, DanaFarber Cancer Institute, Boston, MA, USA; ${ }^{2}$ Department of Biostatistics and Translational Medicine, Medical University of Lodz, Poland; ${ }^{3}$ Postgraduate School of Molecular Medicine, Medical University of Warsaw, Poland; ${ }^{4}$ Department of Cell Biology, Harvard Medical School, Boston, MA, USA; '5epartment of Pathology, Boston Children's Hospital and Harvard Medical School, Boston, MA, USA and ${ }^{6}$ Department of Radiation Oncology, Perlmutter Cancer Center, New York University School of Medicine, New York, NY, USA

* These authors contributed equally to this work

\section{ABSTRACT}

$\mathrm{N}$ coa4 mediates autophagic degradation of ferritin, the cytosolic iron storage complex, to maintain intracellular iron homeostasis. Recent evidence also supports a role for Ncoa4 in systemic iron homeostasis and erythropoiesis. However, the specific contribution and temporal importance of Ncoa4-mediated ferritinophagy in regulating systemic iron homeostasis and erythropoiesis is unclear. Here, we show that Ncoa4 has a critical role in basal systemic iron homeostasis and both cell autonomous and nonautonomous roles in murine erythropoiesis. Using an inducible murine model of Ncoa4 knockout, acute systemic disruption of Ncoa4 impaired systemic iron homeostasis leading to tissue ferritin and iron accumulation, a decrease in serum iron, and anemia. Mice acutely depleted of Ncoa4 engaged the Hif2 $\alpha$-erythropoietin system to compensate for anemia. Mice with targeted deletion of Ncoa4 specifically in the erythroid compartment developed a pronounced anemia in the immediate postnatal stage, a mild hypochromic microcytic anemia at adult stages, and were more sensitive to hemolysis with higher requirements for the Hif $2 \alpha$-erythropoietin axis and extramedullary erythropoiesis during recovery. These studies demonstrate the importance of Ncoa4-mediated ferritinophagy as a regulator of systemic iron homeostasis and define the relative cell autonomous and nonautonomous contributions of Ncoa4 in supporting erythropoiesis in vivo.

Check the online version for the most updated information on this article, online supplements, and information on authorship \& disclosures: www.haematologica.org/content/104/7/1342

(C)2019 Ferrata Storti Foundation

Material published in Haematologica is covered by copyright. All rights are reserved to the Ferrata Storti Foundation. Use of published material is allowed under the following terms and conditions:

https://creativecommons.org/licenses/by-nc/4.0/legalcode. Copies of published material are allowed for personal or internal use. Sharing published material for non-commercial purposes is subject to the following conditions:

https://creativecommons.org//icenses/by-nc/4.0/legalcode, sect. 3. Reproducing and sharing published material for commercial purposes is not allowed without permission in writing from the publisher.

\section{Introduction}

Iron is an essential element for life, required in a diverse set of processes including oxygen binding and transport, ${ }^{1}$ electron transport, ${ }^{2}$ and DNA synthesis and repair., ${ }^{3,4}$ The majority of cellular iron is stored in ferritin, the cellular iron storage complex composed of 24 subunits of ferritin light and heavy chain subunits (FTL, FTH1) that can chelate up to 4,500 iron atoms in a non-toxic ferrihydrite mineral core. ${ }^{5}$ Iron can be released from ferritin via a process termed 'ferritinophagy' ${ }^{6,7}$ FTH1 is bound by NCOA4 (Nuclear Receptor Coactivator 4) and transported to an autophagosome that fuses with a lysosome for ferritin degradation and iron release. ${ }^{8}$ NCOA4-mediated ferritinophagy is responsive to alterations in cellular iron levels to promote ferritinophagy under iron-depleted conditions or decrease ferritinophagy when iron is abundant in the cell. ${ }^{8}$

Given the established importance of NCOA4 in intracellular iron homeostasis, the role of NCOA4 in the response to systemic iron deficiency, and, particularly, its role in the pathophysiology of iron deficiency in anemia, has been of interest. Recent data from in vitro model systems of human, mouse, and zebrafish erythropoiesis support 
a role for NCOA4-mediated ferritinophagy as an important step for iron release prior to mitochondrial iron import and heme biosynthesis. ${ }^{8-11}$ Specifically, perturbation of NCOA4 expression impairs hemoglobinization in the human K562 erythroleukemia and the murine erythropoietic G1E-ER4 cell line models, ${ }^{9}$ suggesting that NCOA4 has a cell autonomous role in erythropoiesis. Constitutive deletion of Ncoa4 in a murine model led to tissue ferritin and iron accumulation as well as a mild hypochromic microcytic anemia, supporting a role for Ncoa4 in both systemic iron homeostasis and erythropoiesis. ${ }^{12}$ The defect in erythropoiesis observed in this model was recently independently confirmed and extended to include a more dramatic anemia in the immediate postnatal period. ${ }^{13}$

Several critical questions regarding in vivo Ncoa4 function remain. First, it is unclear whether a cell autonomous effect accounts for the entirety of the observed anemia phenotype in germline Ncoa4 knockout (KO) mice, or whether the non-erythroid functions of Ncoa4 also contribute to this phenotype. Secondly, Ncoa $4 \mathrm{KO}$ animals likely recruit adaptive mechanisms to compensate for decreased access to ferritin iron stores in the long-term and limit the severity of anemia. ${ }^{12,13}$ To evaluate the cell autonomous role of Ncoa4 in erythropoiesis, we generated a conditional Ncoa $4^{p / f} ; E p o R-$ Cre mouse model with erythroid lineage ablation of Ncoa4. These mice develop a pronounced anemia at the immediate postnatal stage which resolves to a mild hypochromic microcytic anemia in adulthood. However, the anemia, whether in the neonate or adult, is less severe than the anemia that develops in a germline Ncoa4 KO. To examine the adaptive response to $\mathrm{Ncoa} 4$ depletion, we generated a tamoxifen-inducible $N$ coa4 ${ }^{f / p} ; U B C$-cre/ERT2 model to control temporally the somatic depletion of Ncoa4. We found that Ncoa4 deletion in the adult mouse leads to acute anemia followed by a compensatory recovery. Furthermore, this phenotype is more pronounced under conditions of chemically induced stress erythropoiesis. In toto, these data support a model for both cell autonomous and nonautonomous functions of $\mathrm{Ncoa} 4$ in regulating basal and stress erythropoiesis.

\section{Methods}

Details of the experimental procedures are available in the Online Supplement. Primer and short hairpin (sh) RNA sequences are listed in Online Supplementary Table S11.

\section{Mouse models and analysis}

$\mathrm{Ncoa}^{4 / / / 1}$ mice were generated by insertion of loxP sites flanking exons 2 through 6 . The NCOA4 $4^{p / 1 /}$ mouse strain is deposited and available at the Jackson Laboratory (JAX\#033295 Ncoa4 $4^{\mathrm{f}}$ ). Ncoa $4^{\mathrm{n} / \mathrm{f}}$ mice on a C57BL/6 (B6) background were crossed to B6.Cg ${ }^{T_{B S}(\mathrm{BBC} \text { - }}$

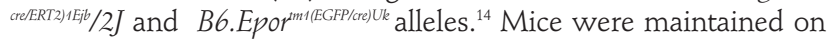
the Prolab Isopro RMH diet (380 ppm iron). Ncoa4 recombination was induced in Ncoa4 $4^{f / 7}$; UBC-cre/ERT2 mice with five doses of 200 $\mathrm{mg} / \mathrm{kg}$ tamoxifen. Hemolysis was induced with $40 \mathrm{mg} / \mathrm{kg}$ phenylhydrazine (PHZ). All experiments were done in adult mice (more than 8 weeks of age, age-matched within the colony) except those on postnatal bleeding (day 10).

\section{Polymerase chain reaction genotyping}

Genomic DNA was extracted from tissues or sorted Ter119+ red blood cells (RBC) and targeting was determined by polymerase chain reaction (PCR).

\section{Hematologic and iron parameters}

Blood was drawn by retro-orbital or submandibular bleeding (serial bleed, $35 \mu \mathrm{L}$ ) for determination of complete blood counts. The method of collection was kept consistent within the same time-course experiment. Serum was obtained from blood drawn by intracardiac bleeding. Serum erythropoietin (R\&D MEP00B) and Ftl (Abcam, ab157713) levels were determined by enzymelinked immunosorbent assays. Tissue non-heme iron concentrations were determined as described previously. ${ }^{15}$ Serum iron levels were determined using a kit (Fisher:23666320).

\section{Flow cytometry}

Bone marrow or spleen cells were stained with anti-Ter119 anti$\mathrm{Cd} 44$ antibodies. Stages were determined based on published methods. ${ }^{16,17}$

\section{Reactive oxygen species measurements}

RBC were incubated with 2,7-dichlorofluorescin diacetate (Sigma:D6883) and analyzed using a Beckman Coulter Cytoflex.

\section{Quantitative polymerase chain reaction}

Quantitative real-time PCR was performed on isolated mRNA using SYBR Green, Actb was used as an internal control.

\section{Western blot analysis}

Cells or tissues were lysed in RIPA buffer. Protein was separated on sodium dodecylsulfate polyacrylamide gel electrophoresis gels and transferred to a membrane. Antibodies to the following compounds (and their sources) were used: Ncoa4 (Santa Cruz Biotechnology sc-373739, 1:100), Fth1 (Cell Signaling:\#4393), Fpn (Alpha Diagnostic International MTP11-A, 1:1000), Actb (Sigma A5441, 1:3000), Hif-2 $\alpha$ (Novus NB100-122, 1:100), Bach1 (Santa Cruz Biotechnology sc-27121, 1:100), Hri (Millipore Sigma 07-728, 1:1000), Eif2 $\alpha$ (Cell Signaling \#9722, 1:1000), Eif2 $\alpha-P$ (Cell Signaling \#9722, 1:100), anti-rabbit IgG (H1L) horse radish peroxidase conjugate (Thermo 31460, 1:3000), and anti-mouse $\operatorname{IgG}(\mathrm{H} 1 \mathrm{~L})$ horse radish peroxidase conjugate (Promega W4021, $1: 7000)$.

\section{Histology}

Tissues were processed as previously described, ${ }^{18}$ fixed in formalin and embedded in paraffin. After deparaffinization, the primary antibody was incubated followed by the secondary antibody and then developed by $3,3^{\prime}$-diaminobenzidine. Antibodies: Fth1 (Cell Signaling:\#4393) and Cd68 (Abcam:ab31630). For quantification of the number of erythroblastic islands, more than five fields (magnification: 10x) per mouse were analyzed and fields were averaged per mouse. Significance was determined by comparing the average of the control group versus that of the $\mathrm{KO}$ group. Islands were identified by the presence of groups of nucleated RBC shown by hematoxylin staining. For quantification of immunohistochemical staining, CD68 was quantified using five fields (magnification: 10x) from each mouse. The percentage of the positive area was calculated by highlighting positively stained regions and averaging them for each mouse.

\section{Lentivirus-mediated short hairpin RNA}

Lentiviral-mediated shRNA knockdown was performed as previously described. ${ }^{19}$

\section{Quantitative proteomics}

Quantitative mass spectrometry-based proteomic analyses were performed as previously described. ${ }^{19,20}$ Briefly, $50 \mu \mathrm{g}$ of digested peptides from each sample were labeled with tandem mass tag reagents and combined at a 1:1:1:1:1:1:1:1:1:1 ratio. Data 
were collected using an Orbitrap Fusion Lumos mass spectrometer. $^{21}$ Mass spectra were processed using a Sequest-based in-house software pipeline. ${ }^{20}$

\section{Bioinformatic analysis}

Gene set enrichment analyses (GSEA) were performed as described elsewhere. ${ }^{22}$ A dataset from Gautier et al. ${ }^{23}$ provided proteomic data for seven stages of erythropoiesis.

\section{Statistics}

No statistical methods were used to predetermine sample size. For comparisons between two groups, a Student $t$-test (unpaired, 2-tailed) was performed for all experiments except for the Student t-tests (unpaired, 1-tailed) in Figures 3C, 4G and Figure 5G (hematocrit, day 2). Results from groups were considered statistically different when $P<0.05$.

\section{Study approval}

All animal studies were conducted under an approved DanaFarber Cancer Institute Institutional Animal Care and Use Committee (IACUC) protocol (15-020).

\section{Results}

\section{Temporally induced systemic Ncoa4 loss in the adult leads to defective iron mobilization and anemia}

To assess the effects of deletion of Ncoa4 in the adult animal, we generated a conditional Ncoa4 KO model (Ncoa4t ${ }^{t r i}$, Online Supplementary Figure S1A). We crossed these animals with the UBC-cre/ERT2 allele to generate a temporally inducible Ncoa4 ${ }^{t / p /} ; U B C$-cre/ERT2 systemic KO model (hereafter $\mathrm{Ncoa}^{\mathrm{rec}}$ with tamoxifen administration). One week after tamoxifen administration, there was efficient recombination of the Ncoa4 locus and corresponding depletion of Ncoa4 protein (Figure 1A, Online Supplementary Figure S1B,C). Acute depletion of Ncoa4 impaired ferritinophagy resulting in varying degrees of tissue accumulation of Fth1 (Figure 1B, Online Supplementary Figure S1D). Fth1 accumulation correlated with increased iron retention in the spleen and liver of Ncoa4 $4^{\text {rcc }}$ mice (Figure 1C,D) although no differences were observed in the bone marrow (Figure 1E). There was no significant difference in serum ferritin levels in control versus Ncoat ${ }^{\text {rec }}$ mice (Online Supplementary Figure S1E). Consistent with a decrease in tissue ferritin turnover and decreased cellular iron release, serum iron was decreased after Ncoa4 deletion (Figure 1F). Ferroportin (Fpn) was increased in the spleen and liver and to a lesser extent in the duodenum (Figure 1G) of Ncoa4t mice, suggesting a compensatory response to low serum iron to increase iron export from tissues to support erythropoiesis. Despite the acute decrease in serum iron levels, no differences were observed in liver hepcidin expression (Online Supplementary Figure S1F). Taken together, these results suggest that ferritinophagy is important for maintaining serum iron levels. However, owing to systemic KO, the tissue source(s) of iron mobilized into the serum as a consequence of ferritinophagy is unclear.

To evaluate the effects of a temporally induced block in ferritinophagy on erythropoiesis, we analyzed complete blood counts in Ncoa4 ${ }^{p / p /}$ and Ncoart $4^{r c c}$ mice (Figure $1 \mathrm{H}$, Online Supplementary Table S1) 7 days after the last tamoxifen administration. Ncoa 4 deletion resulted in a drop in RBC number, hematocrit and hemoglobin (Figure 1H). No significant differences were observed in mean corpuscular volume or red cell distribution width, likely due to the acuteness of the model (Figure 1H). The phenotype was independent of gender and not solely due to the administration of tamoxifen or the UBC-Cre/ERT2 allele (Online Supplementary Tables S1-3).

As Ncoa 4 is highly expressed at the orthochromatic erythroblast stage, ${ }^{24}$ we evaluated the effects of Ncoa4 depletion on erythroid differentiation using flow cytometric analysis of Ter119 and Cd44 in bone marrow precursors. ${ }^{17}$ Ncoa4 depletion had no impact on erythroid differentiation through the reticulocyte stage (Online Supplementary Figure $S 1 G$ ), similarly to a previously described model of germline Ncoa4 deletion. ${ }^{12}$

Given the acute anemia, we next analyzed mice for phenotypic and molecular markers of a recovery response to anemia. Ncoa4t ${ }^{r c c}$ mice showed increased erythropoietin (Epo) levels in serum (Figure 1I) and increased hypoxiainduced factor-2 $\alpha$ (Hif-2 $\alpha$ ) expression in the kidney (Figure 1J) indicating an appropriate compensatory response to anemia. ${ }^{25-27}$ However, spleen size, a marker of stress erythropoiesis, was not different (Figure 1K). On the other hand, Bach1, Hri and Eif2 $\alpha-P$ were upregulated in Ncoa ${ }^{\text {rc }}$ mice (Figure 1L). This is consistent with activation of a transcriptional program in erythroblasts of Ncoa4 KO mice which would limit globin synthesis when heme levels are low. ${ }^{28,29}$ Collectively, our data demonstrate a dynamic role for Ncoa4 in systemic iron homeostasis with constant flux through the ferritinophagy pathway under basal conditions which supports erythropoiesis.

\section{Anemia induced by loss of Ncoa4 in the adult leads to a compensatory response over time}

To evaluate the effects of acute Ncoa4 deletion over the lifespan of a murine RBC, we induced Ncoa4 recombination and performed serial complete blood counts. We observed the expected anemia immediately following depletion (day 11) (Figure 2A). However, over a period of 5 weeks, the hematocrit and hemoglobin values recovered to levels comparable to those of control animals (Figure 2A). Conversely, reticulocyte hemoglobin content and mean corpuscular volume were both significantly decreased, consistent with a compensated microcytosis (Figure 2A). The normal hematocrit and hemoglobin levels are in contrast to the constitutive model of Ncoa4 depletion in which hematocrit and hemoglobin were decreased, suggesting differences in the outcome of an adaptive response to Ncoa4 deletion in adult animals versus life-long Ncoa4 loss. Nevertheless, similarly to constitutive knockout mouse models ${ }^{12,13}$ we found no differences in erythrocyte precursor differentiation in the adult animal (Online Supplementary Figure S2A).

At the end point of the experiment, Ncoat ${ }^{\text {rac }}$ mice showed Fth1 accumulation in tissues, including those not affected by acute Ncoa4 depletion (pancreas, Figure 2B,C). In contrast to acute deletion of Ncoa4, serum iron levels were increased in long-term Ncoatre mice (Figure 2D), which is in line with results from a constitutive model of Ncoa4 deletion. ${ }^{12}$ This may be a consequence of defective ferritinophagy leading to tissue iron overload and shunting of intracellular iron to export pathways or consequent to sustained mobilization of iron induced by anemia. To evaluate the former, we analyzed Fpn expression in several tissues. After long-term Ncoa4 ablation, we observed normalization of Fpn levels in spleen and liver compared 
to the levels after acute depletion (Figure 2E). This normalization is consistent with an increase in hepcidin levels (Figure 2F), increase in serum iron and resolution of anemia. However, Fpn levels were sustainably increased in the duodenum as part of a compensatory response to iron deficiency and, in part, explaining the high iron in serum. It is to be expected that duodenal Fpn expression would decrease with a longer period of time as demonstrated in

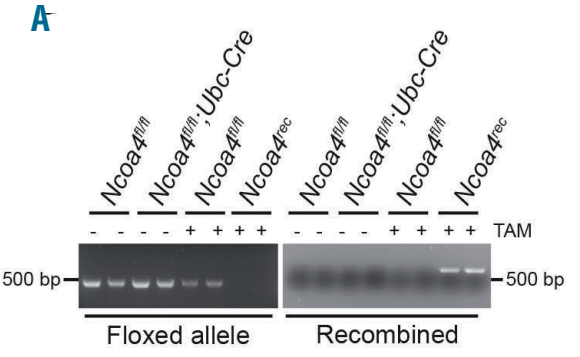

B

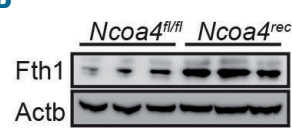

LIVER
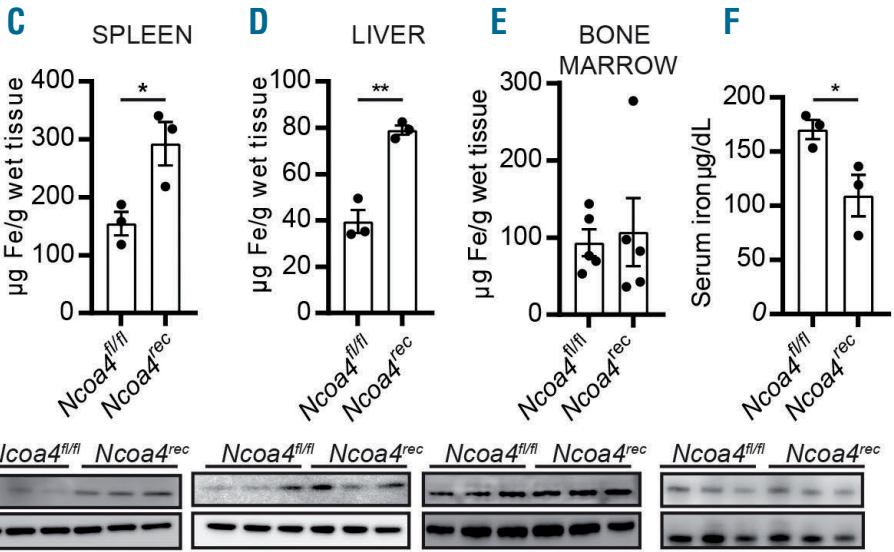

BRAIN
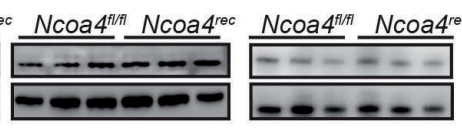

KIDNEY

PANCREAS

BONE MARROW

G

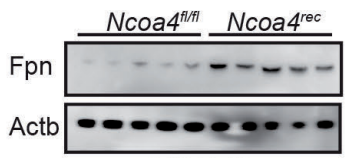

SPLEEN

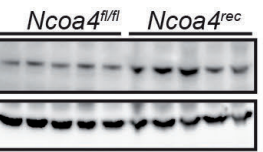

LIVER

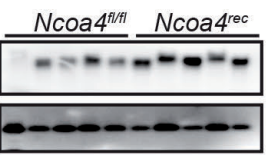

DUODENUM

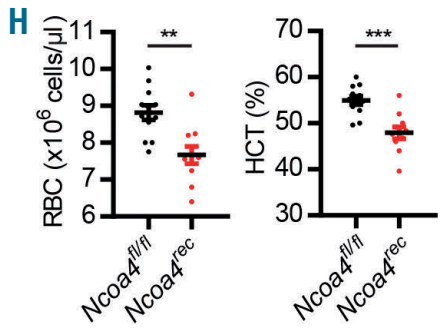

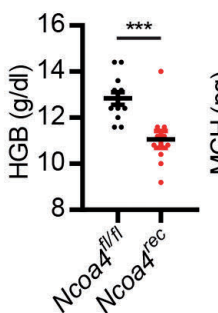
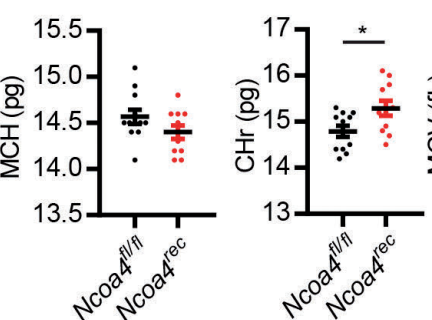

$J$$$
\text { I }
$$



Ncoa4t/f/l Ncoa4rec

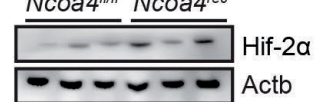

KIDNEY

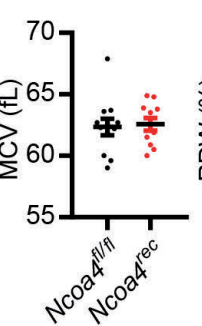

K.

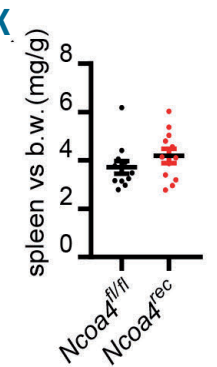

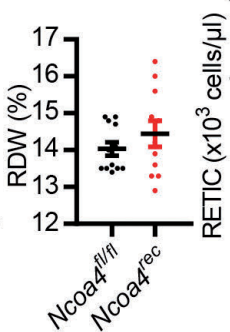

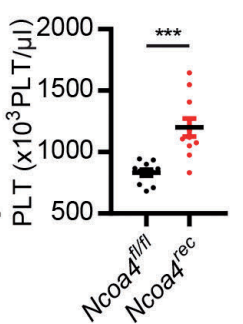

L

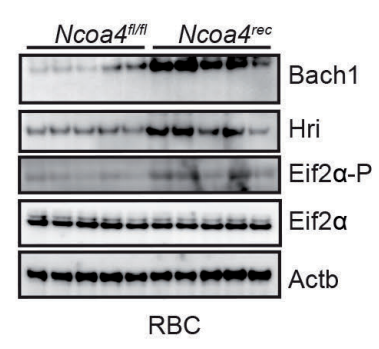

Figure 1. Acute systemic knockout of Ncoa4 impairs ferritinophagy and erythropoiesis. A murine model of Ncoa4 deficiency was generated using a tamoxifen-

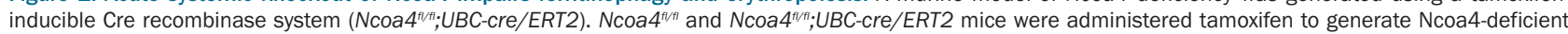

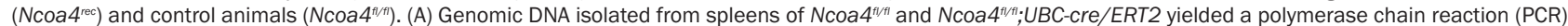
product at $470 \mathrm{bp}$ when using primers directed at the floxed allele (left panel, see Methods). This band was not detected in Ncoa $4^{\text {rec }}$ mice. Using primers directed at the recombined allele, a PCR product of 540 bp was detected for Ncoa $4^{\text {rec }}$ but not Ncoa $4^{\text {ft/ll }}$ (right panel). (B) Increased Fth1 protein levels in liver, spleen, kidney, brain but not pancreas or bone marrow from Ncoa $4^{\text {rec }}$ mice 11 days after initiation of tamoxifen administration. $\beta$-actin (Actb) served as a loading control. (C-E) Tissue iron levels in spleen (C), liver (D) and bone marrow (E) from Ncoa $4^{\text {rec }}$ in comparison to Ncoa $4^{4 / f i}$ mice [3-5 mice/group, error bars represent the standard error of mean (s.e.m.)] (F) Lower serum iron levels in Ncoa $4^{\text {rec }}$ mice (3 mice/group, error bars represent the s.e.m.). (G) Increased Fpn protein levels in spleen, liver, and duodenum from Ncoa $4^{\text {rec }}$ mice 11 days after initiation of tamoxifen administration. Actb served as a loading control. (H) Whole blood from adult (>12 weeks) Ncoa4 $4^{\text {fl/t }}$ and $N \mathrm{Nco}^{\mathrm{rec}}$ mice 7 days after the last administration of tamoxifen was acquired by retro-orbital bleeding for complete blood count profiling $\left(\mathrm{Ncoa} 4^{\mathrm{f} / \mathrm{fl}}\right.$, $\mathrm{n}=12 ; \mathrm{Ncoa} 4^{\mathrm{rec}}$ $\mathrm{n}=11$, error bars represent the s.e.m.). (I) Elevated erythropoietin levels in serum of Ncoa ${ }^{\text {rec }}$ mice ( 8 mice/group, error bars represent the s.e.m.). (J) Increased Hif$2 \alpha$ protein levels in kidney from Ncoa4 ${ }^{\text {rec }}$ mice 11 days after initiation of tamoxifen administration. Actb served as a loading control. (K) No significant change in spleen size of $\mathrm{Ncoa}_{4}^{\mathrm{f} / \mathrm{ft}}$ and $\mathrm{Ncoa} 4^{\mathrm{rec}}$ male and female mice 11 days after the initiation of tamoxifen administration (4 males and 7 females/group). (L) Bach1, Hri, Eif $2 \alpha$ and Eif $2 \alpha-P$ protein levels in red blood cells of $N$ coa $4^{\mathrm{t} / \mathrm{fl}}$ mice and $\mathrm{Ncoa} 4^{\mathrm{rec}}$. Actb served as a loading control. For all panels, statistical comparison was performed using a two-tailed Student $t$-test: $* P<0.05, * * P<0.01, * * * P<0.001$. TAM: tamoxifen; Fth1: ferritin heavy chain 1; Fpn: ferroportin; RBC: red blood cell count; HCT: hematocrit; HGB: hemoglobin; $\mathrm{MCH}$ : mean corpuscular hemoglobin; $\mathrm{CHr}$ : reticulocyte hemoglobin content; MCV: mean corpuscular volume; RDW: red blood cell distribution width; RETIC: reticulocyte count; PLT: platelet count; Epo: erythropoietin; b.w.: body weight. 
a constitutive Ncoa4 $\mathrm{KO}$ model, ${ }^{12}$ in which duodenal Fpn expression was elevated in 2 -month old mice but decreased at 6 months.

Alternatively, or in addition, serum iron overload may be a secondary consequence of ineffective erythroid utilization of iron. To examine this, we evaluated the Hif-2 $\alpha$-Epo pathway. As shown in Figure 1I,J, this compensatory mechanism is activated early after induction. Five weeks later, we observed a trend towards an increase in Epo levels in Ncoa $4^{\text {rec }}$ mice (Figure 2G) which correlated with sustained overexpression of Hif- $2 \alpha$ in the kidneys (Figure $2 \mathrm{H}$ ). As in the early response, longer-term Ncoa 4 deletion had no effect on spleen size (Online Supplementary Figure S2B). Taken together, these data show that Ncoa4 depletion induces an acute, uncompensated anemia that normalizes over time and achieves a new steady state in which the Hif- $2 \alpha$-Epo axis response induces erythropoiesis sufficient to overcome the hemoglobin deficit by virtue of producing more $\mathrm{RBC}$ that are poorly hemoglobinized. In the setting of adequate serum iron, which is achieved by increased iron export from tissues and iron import from the diet, the persistent cellular hemoglobin deficit suggests a defect intrinsic to the $\mathrm{RBC}$ itself, specifically that the erythroid precursor is unable to mobilize sufficient iron for heme synthesis. ${ }^{9}$

The stress-erythropoietic response to hemolysis is less effective in maintaining erythrocyte production in Ncoa4-deficient mice

To evaluate the role of Ncoa4 under conditions of stress erythropoiesis, we induced hemolysis with $\mathrm{PHZ}$ (Online
A

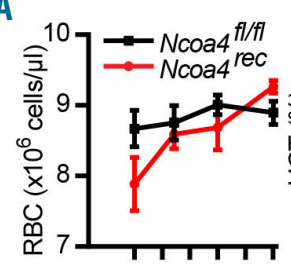

Days 1180

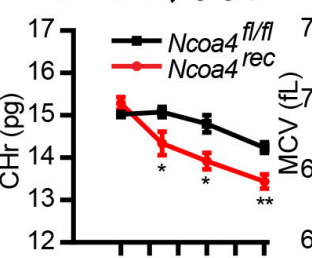

Days 1180.232096

C

Ncoa4 $4^{\mathrm{H} / \mathrm{t}}$

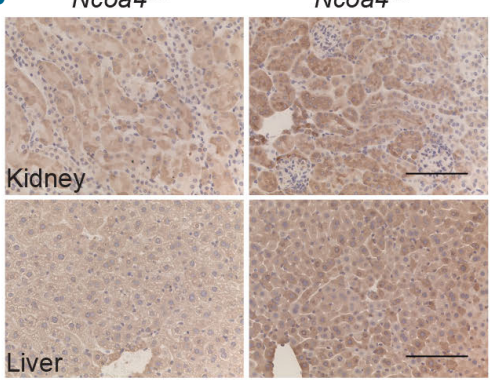

Ncoatrec

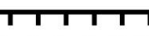

1 18253096

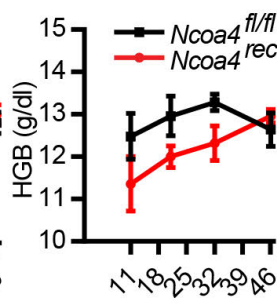

15.5
$15.0-$
$\widehat{D}_{2} 14.5-$
$\mathrm{I}_{1} 14.0-$
$\sum_{13.5}$
13.0

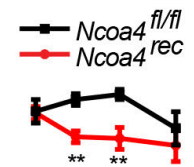

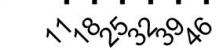

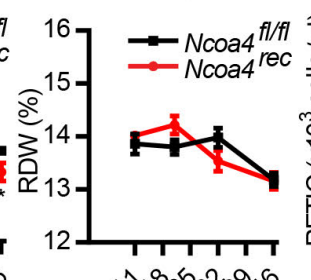

1,8053296

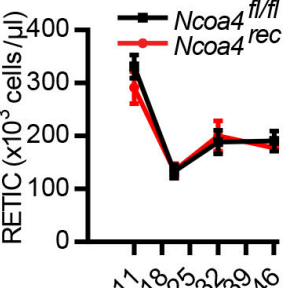

1,80532096

D

B

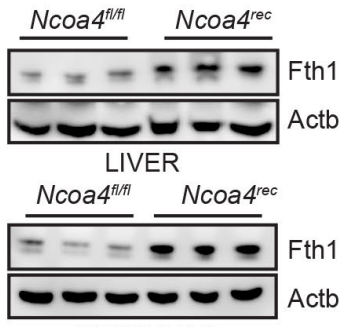

PANCREAS

F
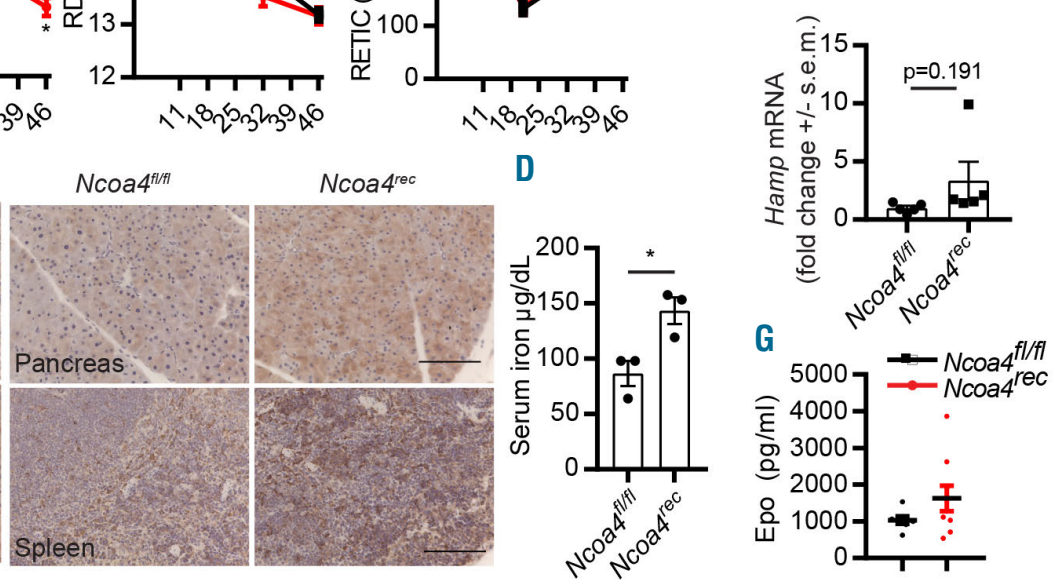

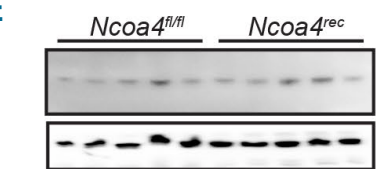

SPLEEN

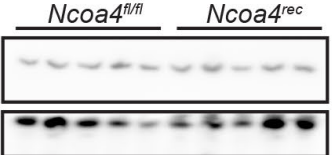

LIVER

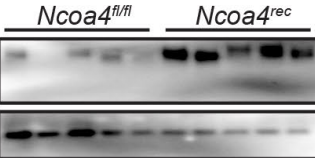

DUODENUM
H Fpn Actb

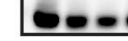

$00-0$

Figure 2. Prolonged Ncoa4 deficiency produces a microcytic hypochromic anemia. Ncoa $4^{f / f t} ; U B C-c r e / E R T 2$ and $N c o a 4^{f / / f l}$ mice were treated with tamoxifen and whole blood was analyzed serially and tissue and serum at the endpoint. (A) Whole blood from Ncoa $4^{f / f i}$ and $N$ coa $4^{\text {rec }}$ mice was obtained by submandibular bleeding and analyzed at weekly intervals after tamoxifen administration for parameters of the complete blood count [5 mice/group, error bars represent the standard error of mean (s.e.m.)]. (B) Increased Fth1 protein levels in liver and pancreas from Ncoa $4^{\text {rec }}$ mice 46 days after initiation of tamoxifen administration. $\beta$-actin (Actb) served as a loading control. (C) Increased Fth1 staining in kidney, liver, spleen and pancreas of Ncoa $4^{\text {rec }}$ mice 46 days after initiation of tamoxifen administration. Representative field $(20 \mathrm{x})$ of 5 mice/group (scale bar $=100 \mu \mathrm{m}$ ) (D) Elevated serum iron levels in Ncoa $4^{\text {rec }}$ mice in comparison to $N c o a 4^{t / f t}$ mice at the endpoint. ( 3 mice/group, error bars represent the s.e.m.). (E) Fpn protein levels in spleen, liver, and duodenum from Ncoa $4^{\text {rec }}$ mice 46 days after initiation of tamoxifen administration. Actb served as a loading control. (F) Hamp mRNA fold-change in livers of Ncoa4 ${ }^{\text {rec }}$ and Ncoa $4^{t / f l}$ mice ( $n=5 /$ group, error bars represent the s.e.m.). (G) Erythropoietin levels in serum of $\mathrm{Ncoa}^{\mathrm{ft} / \mathrm{fl}}(\mathrm{n}=7)$ versus Ncoa $4^{\text {rec }}(n=9)$ mice at day 46 after the initiation of tamoxifen administration (error bars represent the s.e.m.). $(\mathrm{H})$ Increased Hif- $2 \alpha$ protein levels in kidneys from Ncoa $4^{\text {rec }}$ mice 46 days after the initiation of tamoxifen administration. Actb served as a loading control. For all panels, statistical comparison was performed using a two-tailed Student $t$-test: $* P<0.05, * * P<0.01, * * * P<0.001$. RBC: red blood cell count; HCT: hematocrit; HGB: hemoglobin; $\mathrm{MCH}$ : mean corpuscular hemoglobin; CHr: reticulocyte hemoglobin content; MCV: mean corpuscular volume; RDW: red blood cell distribution width; RETIC: reticulocyte count; PLT: platelet count; Fth1: ferritin heavy chain 1; Fpn: ferroportin; Epo: erythropoietin. 
Supplementary Figure 2SC). Ncoa4 ${ }^{\text {rec }}$ mice reached lower RBC numbers, hematocrit and hemoglobin nadirs which required a longer time to recover than those of $N$ coa $4^{\text {f/f }}$ mice (Figure 3A, Online Supplementary Figure S2D) although they did reach the same levels as those of controls at a later time-point (Online Supplementary Table S4). Reticulocyte percentage was lower in $\mathrm{KO}$ mice at initial time points after $\mathrm{PHZ}$ suggesting an initial sluggish reticulocytosis response to PHZ-induced anemia (Figure 3B). However, $\mathrm{KO}$ animals demonstrated a higher reticulocyte peak at day 10 and percentage reticulocyte level remained significantly elevated compared to that of controls at the endpoint (day 12). The trend observed in absolute reticulocyte number (Online Supplementary Figure S2D) was the same as the reticulocyte percentage, indicating that the increase in reticulocytosis observed is not a consequence of decreased RBC number due to increased fragility of the cells.

Both Epo (Figure 3C) and Hif-2 $\alpha$ (Figure 3D) levels were elevated in mutant animals, which also had enlarged

A

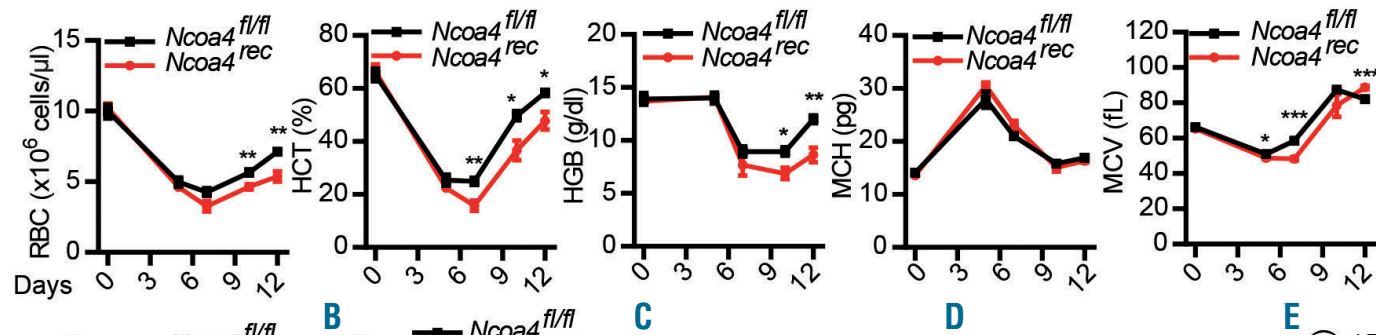

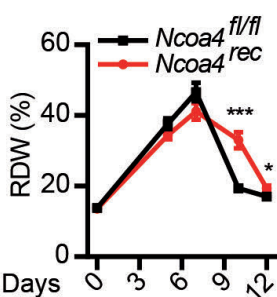

F

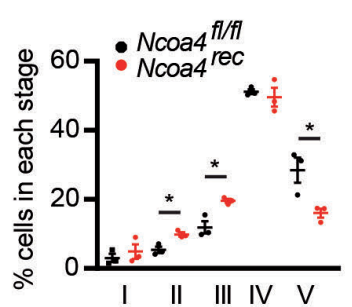

H

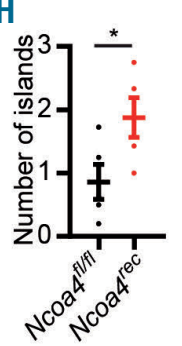

I



G

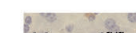

C

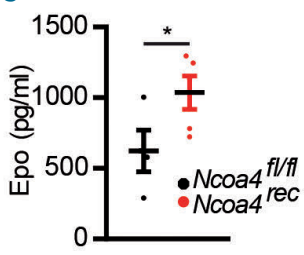

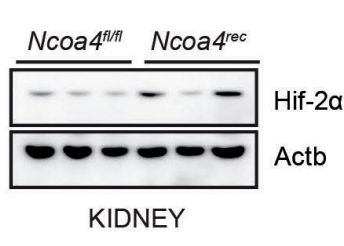

K

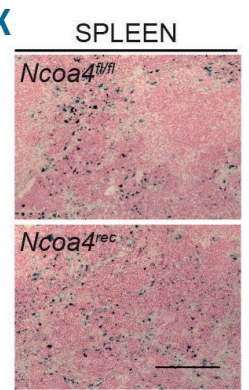

J

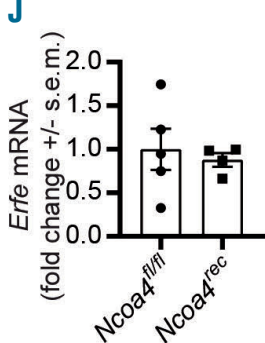

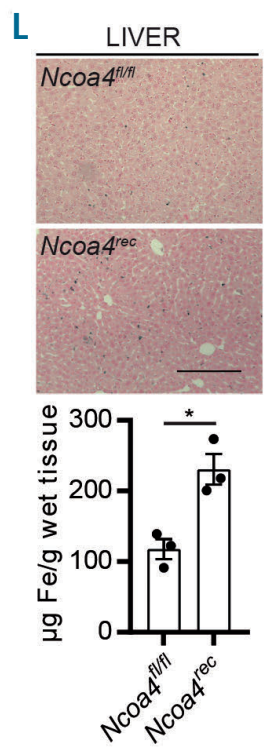
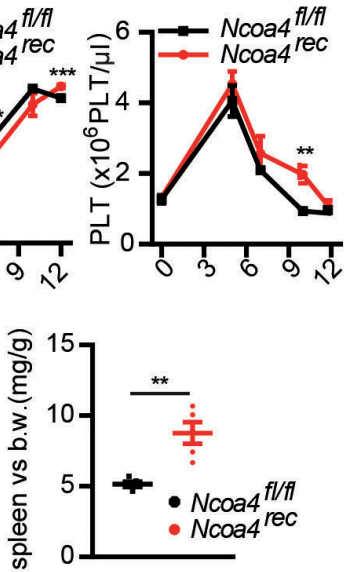
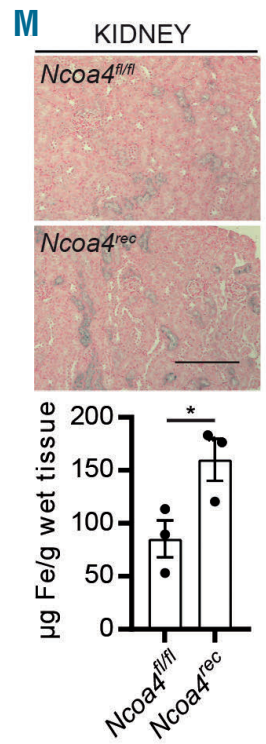

Figure 3. Ncoa4-deficient mice show a less effective response to induced stress-erythropoiesis. Ncoa $4^{\text {rec }}$ and Ncoa $4^{f / t / t}$ mice were treated with phenylhydrazine to induce red blood cell lysis. Whole blood, tissue, and serum were analyzed. Panels C-M show measurements performed at the experimental endpoint (day 12 ), 8 days after the last administration of phenylhydrazine. (A) Serial complete blood count profiling of Ncoa $4^{\text {rec }}$ and Ncoa $4^{f / f l}$ mice [5 mice/group, error bars represent the standard error of mean (s.e.m)]. (B) Reticulocyte percentage from Ncoa $4^{\text {rec }}$ and Ncoa $4^{t / f}$ mice (5 mice/group, error bars represent the s.e.m). (C) Erythropoietin levels in serum of $\mathrm{Ncoa}_{4}^{f / H}(\mathrm{n}=4)$ versus Ncoa4 ${ }^{\text {rec }}(\mathrm{n}=5)$ mice (error bars show the s.e.m). (D) Increased Hif- $2 \alpha$ protein levels in kidney from Ncoa4 ${ }^{\text {rec }}$ mice after PHZ administration. $\beta$-actin (Actb) served as a loading control. (E) Spleen size normalized to body weight is increased in Ncoa $4^{\text {rec }}$ mice at the endpoint (5 mice/group, error bars represent the s.e.m). (F) Ter119-Cd44 staining of bone marrow cells isolated from Ncoa $4^{\text {fl/t }}$ and Ncoa4 ${ }^{\text {rec }}$ mice. Cells within the Ter119 ${ }^{+}$population were quantified and cells at each stage of differentiation are represented as the percentage of the total population ( 3 mice/group, error bars represent the s.e.m.) Stage I: proerythroblasts, stage II: basophilic and polychromatic erythroblasts, stage III: orthochromatic erythroblasts, stage IV: reticulocytes and stage V: mature red cells. (G) Representative field (20x) of 5 mice/group (scale bar $=100 \mu \mathrm{m}$ ) of Cd68 staining in liver from Ncoa4 ${ }^{t / / f}$ and $N$ coa $4^{\text {rec }}$ mice. (H) Quantification (at least 5 fields per sample) of erythroblastic islands ( 5 mice/group, error bars represent the s.e.m.) associated with Cd68 staining. (I) Hamp mRNA fold-change in livers of Ncoa4 ${ }^{\text {rec }}$ and


error bars represent the s.e.m.). (K-M) Increase in tissue iron staining and levels in Ncoa $4^{\text {rec }}$ mice as determined by Prussian blue staining of spleen (K), liver (L) and kidney (M) (representative field of 5 mice/group, 10x, scale bar=200 $\mu \mathrm{m})$. Bottom: Tissue iron levels in spleen, liver, and kidney (3 mice/group, error bars represent the s.e.m.). Statistical comparison was performed using a two-tailed Student $t$-test: $* P<0.05, * * P<0.01, * * * P<0.001$, except for panel B, where a one-tailed Student $t$-test was performed: ${ }^{*} P<0.05$. RBC: red blood cell count; HCT: hematocrit; HGB: hemoglobin; MCH: mean corpuscular hemoglobin; CHr: reticulocyte hemoglobin content; MCV: mean corpuscular volume; RDW: red blood cell distribution width; RETIC: reticulocyte count; PLT: platelet count; Epo: erythropoietin; b.w.: body weight. 
spleens and an increase in erythrocyte precursors when euthanized prior to complete recovery (Figure 3E,F). However, unlike a previously reported germline mutant maintained on a low iron diet, there was no block in differentiation at the orthochromatic erythroblast stage, ${ }^{12}$ indicating that different erythropoietic stresses affect the Ncoa4 ${ }^{\text {rec }}$ phenotype differently.

Splenic macrophages play a key role in making iron available for erythropoiesis by phagocytosing senescent $\mathrm{RBC}$ and recycling iron from heme. ${ }^{30}$ Consistent with enlarged spleen size and activation of the Epo pathway, Ncoa4 deletion increased spleen erythropoiesis in PHZtreated Ncoa4 ${ }^{\text {rec }}$ mice compared to controls (Online Supplementary Figure S2E). In addition, specific populations of transient macrophages in the liver have been implicated in RBC phagocytosis and erythroblastic island formation under conditions of stress erythropoiesis. ${ }^{31-33}$ Indeed, macrophages were detected by Cd68 staining in the livers of $\mathrm{Ncoa}_{4}{ }^{\mathrm{flf} f}$ and $\mathrm{Ncoa}^{\text {rec }}$ mice (Figure $3 \mathrm{G}$ ) and there was a significant increase in the number of erythroblastic islands in the livers of Ncoat ${ }^{\text {rec }}$ mice (Figure $3 \mathrm{H}$, Online Supplementary Figure S2F). This suggests an enhanced reliance on both spleen- and liver-based erythropoiesis for recovery. Interestingly, we observed decreased hepcidin levels in livers of Ncoa4 null mice suggesting an active requirement for mobilization of cytosolic iron to support recovery from anemia (Figure 3I). Of note, erythroferrone (Erfe) levels in bone marrow were unchanged (Figure 3J). According to the kinetics of activation of the Erfe-Hamp pathway $^{34}$ we would expect an increase in Erfe after hemolytic insult coincident with the high Epo/low Hamp levels observed; therefore, these results point towards normalization of Erfe expression during the recovery period. Lower RBC numbers, hematocrit and hemoglobin nadirs in acute Ncoatrec mice treated with PHZ could suggest either delayed engagement of the reticulocytosis machinery and/or increased turnover of RBC or RBC precursors. Data described above appear to support an initial sluggish reticulocytosis followed by robust engagement of compensatory reticulocytosis. One marker of RBC turnover in response to $\mathrm{PHZ}$ is accumulation of hemosiderin deposits in spleen and liver macrophages, likely representing iron from phagocytosed RBC. Perls Prussian blue staining of hemosiderin deposits confirmed higher iron retention in spleen and liver (Figure $3 \mathrm{~K}, \mathrm{~L}$ ) from Ncoa4 ${ }^{\text {rec }}$ mice. Likewise, iron concentration and Fth1 staining were increased in these tissues (Figure 3K, L, bottom panel, Online Supplementary Figure S2G). Another marker of RBC turnover is accumulation of filtered iron in renal proximal tubules. ${ }^{35,36}$ Ncoa $4^{\text {rec }}$ mice accumulated higher levels of iron in the kidney in response to PHZ (Figure 3M). While these accumulations of iron could suggest a higher turnover of $\mathrm{RBC}$, these markers are complicated to interpret in mice with systemic Ncoa4 depletion as a block in ferritinophagy flux will lead to accumulation of tissue iron deposits even in the absence of an increase in $\mathrm{RBC}$ turnover. We also measured haptoglobin and hemopexin levels in serum as measures of RBC lysis. Haptoglobin and hemopexin levels were similarly downregulated in Ncoa $4^{\text {rec }}$ and control animals, likely due to massive PHZ-induced RBC lysis (Online Supplementary Figure S2H). Given the complexity of the model, the relative contribution of sluggish reticulocytosis or increased RBC turnover is unclear and supports investigation of the phenotype in model systems with targeted Ncoa4 deletion.

\section{Ncoa4 has a cell autonomous role in erythropoiesis}

To evaluate the cell autonomous role of Ncoa4 in erythropoiesis in vivo, we crossed Ncoa4t/fl animals with the erythropoietin receptor EpoR-Cre allele which results in erythroid-specific deletion in mid-gestation. ${ }^{14}$ Ter119 ${ }^{+}$-specific efficient Ncoa4 recombination was demonstrated in Ncoa4 ${ }^{f / f} ;$ EpoR-Cre mice (Online Supplementary Figure S3A,B). Adult Ncoa4 ${ }^{f l / f}$;EpoR-Cre animals had a mild hypochromic microcytic anemia compared to EpoR-Cre mice, with decreased hemoglobin, hematocrit, mean corpuscular hemoglobin, reticulocyte hemoglobin content, and mean corpuscular volume (Figure 4A, Online Supplementary Table S5). Spleen size was unchanged compared to that of controls (Online Supplementary Figure S3C). Epo levels were downregulated in $\mathrm{KO}$ mice (Figure 4B); however, discordant with the Epo levels, Hif-2 $\alpha$ kidney protein levels were slightly elevated (Figure 4C). Correlating with Ncoa4 depletion in the erythroid compartment, Fth1 (Figure 4D) and iron levels (Figure 4E) were elevated in bone marrow from $N c o a 4^{f / /} ; E p o R-C r e$ mice. This increase was in contrast to the lack of Fth1 accumulation in the acute depletion model (Figure 1B, E) likely due to long-term Ncoa4 depletion. This is consistent with Fth1 accumulation in the bone marrow of mice with constitutive systemic deletion of Ncoa $4 .{ }^{12}$ Consistent with an anemic phenotype, Bach1 and Eif2 $\alpha$-P levels were elevated in RBC from KO mice (Figure 4F). Similarly to the findings in adult systemic $\mathrm{KO}$ mice, there were no differences in differentiation of bone marrow precursors at any stage (Online Supplementary Figure S3D). Germline Ncoa4 null mice are severely anemic in the immediate postnatal period. ${ }^{13}$ We found that erythroid-specific deletion of Ncoa4 similarly leads to a more dramatic phenotype when we analyzed complete blood counts from mice at postnatal day 10 (P10). P10 Ncoa4 $4^{f / f} ;$ EpoR-Cre mice were anemic in comparison to control mice with significant decreases in RBC numbers, hematocrit and hemoglobin (Figure 4G, Online Supplementary Table S6). However, the Ncoa4 ${ }^{\text {fl/ }}$;EpoR-Cre P10 anemia was less severe than the postnatal anemia observed in mice with germline Ncoa4 loss. ${ }^{13}$ Overall, these findings support a cell autonomous role for Ncoa4 in erythropoiesis which is more severe in the postnatal period, but this phenotype is milder than induced systemic (Figure $1 \mathrm{H}$ ) or germline Ncoa4 depletion, ${ }^{12}$ suggesting a concurrent, non-cell autonomous role for Ncoa4 in supporting erythropoiesis.

We further evaluated the erythroid cell autonomous effects of Ncoa4 depletion during PHZ-induced hemolysis. Adult mice were dosed with PHZ on days 0,1 and 3 and analyzed at day 7. At day 7, RBC numbers, hematocrit, hemoglobin and mean corpuscular hemoglobin

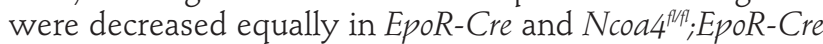
mice (Figure 5A, Online Supplementary Table S7). Despite similar complete blood count parameters 7 days after administration of PHZ, Ncoa $4^{\mathrm{fl} / \mathrm{f}}$; EpoR-Cre mice had elevated serum Epo levels (Figure $5 B$ ) and kidney Hif- $2 \alpha$ protein and mRNA expression (Figure 5C, D) compared to EpoRCre mice, pointing to differential engagement of the Epo system for proportionate erythrocyte recovery. Despite differential increases in Epo levels, PHZ induced an equally potent increase in spleen size (Online Supplementary Figure S3E) and upregulation of erythrocyte precursors in bone marrow and spleen (Online Supplementary Figure $S 3 F)$. There was an increase in erythroblastic islands in the livers of $\mathrm{Ncoa}_{4}^{\mathrm{fl} / \mathrm{l}}$;EpoR-Cre mice (Figure $5 \mathrm{E}$ ) as well as an 
increase in splenic erythropoiesis (Online Supplementary Figure $S 3 G$ ), confirming a differential activation of stress erythropoiesis in these mice. We also noted an increase in $\mathrm{Cd} 8^{+}$macrophages in $\mathrm{Ncoa}^{\mathrm{fl} / \mathrm{f}}$;EpoR-Cre mice (Figure 5F), suggesting liver macrophages are present in these mice for recycling damaged $\mathrm{RBC}$ but also to contribute to stress erythropoiesis. ${ }^{37-39}$ However, there were no apparent differences in liver hepcidin expression (Online Supplementary Figure $S 3 H$ ). To evaluate RBC lysis, we analyzed haptoglobin and hemopexin levels. Here, we observed a trend towards decreased hemopexin in $\mathrm{Ncoa}_{4}^{\mathrm{fth}}$;EpoR-Cre mice which could suggest higher RBC fragility after PHZ administration (Online Supplementary Figure S3I).
Next, we performed a time-course experiment to understand the kinetics of recovery from $\mathrm{PHZ}$-induced anemia (Figure 5G). Similarly to the Ncoa $4^{\text {fl/ }}$; UBC-cre/ERT2 model, Ncoa $4^{f / 7} ; E$ EPoR-Cre mice reached a lower hematocrit nadir (Figure $5 \mathrm{G}$ ). However, within the time course of the experiment, Ncoa $4^{f / f}$;EpoR-Cre mice recovered the same hematocrit levels as EpoR-Cre mice (Figure 5G), suggesting reticulocytosis is not impaired in this model. This is in contrast to the systemic KO mice in which recovery was incomplete in the same time period suggesting that non-erythroid loss of Ncoa4 impairs recovery, likely through disrupting systemic iron metabolism. At the endpoint of the time-course experiment (day 11 after PHZ), spleen size
A
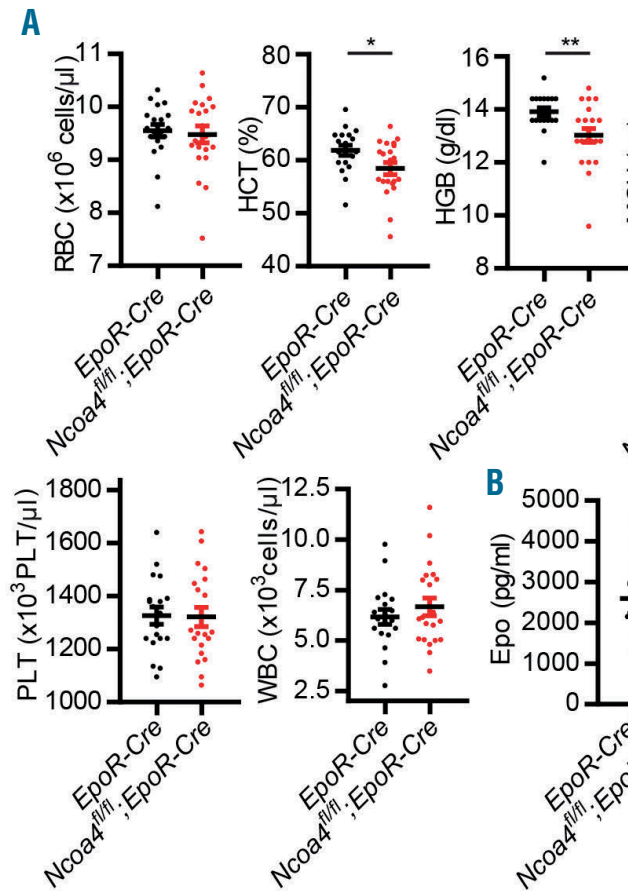

B $5000 \div \approx$ C

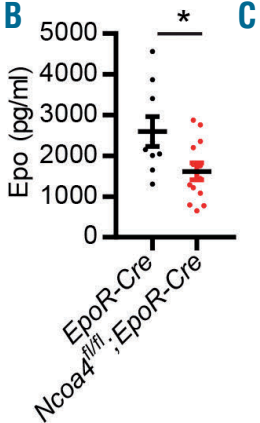

C
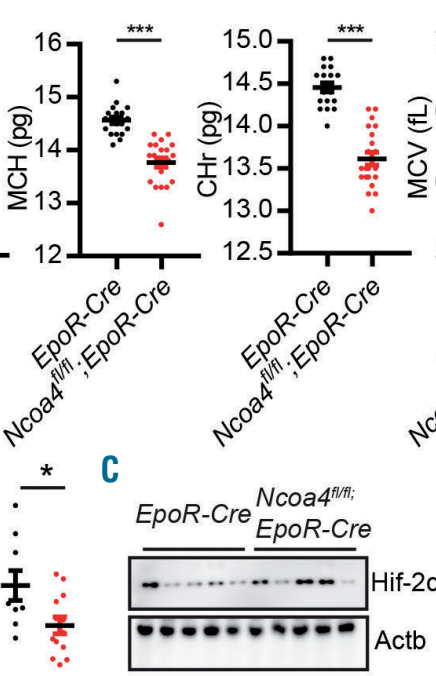

KIDNEY

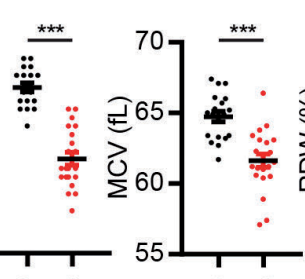

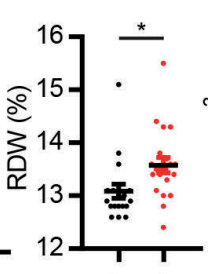
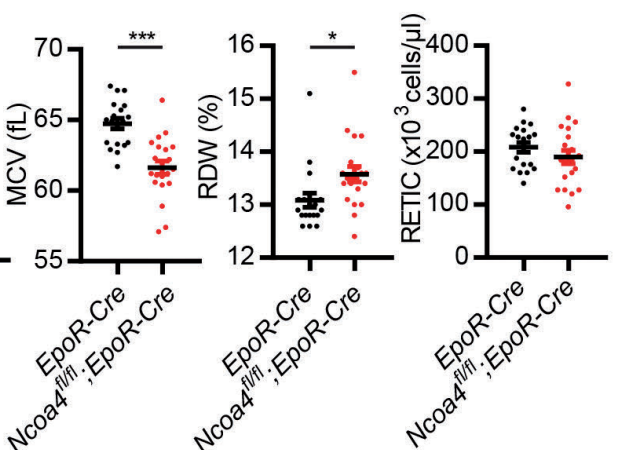

D
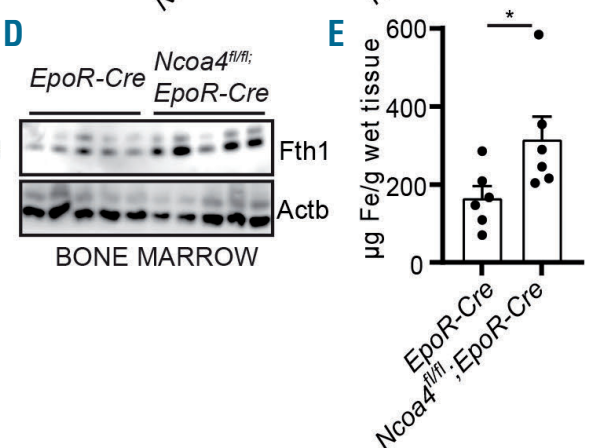

$\mathrm{F}$

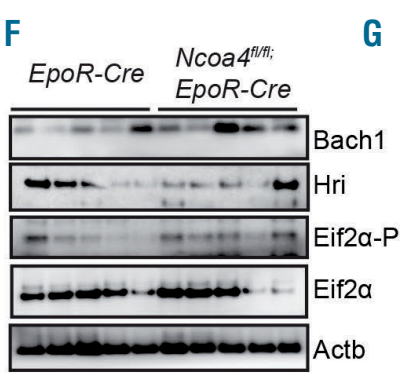

$\mathrm{RBC}$
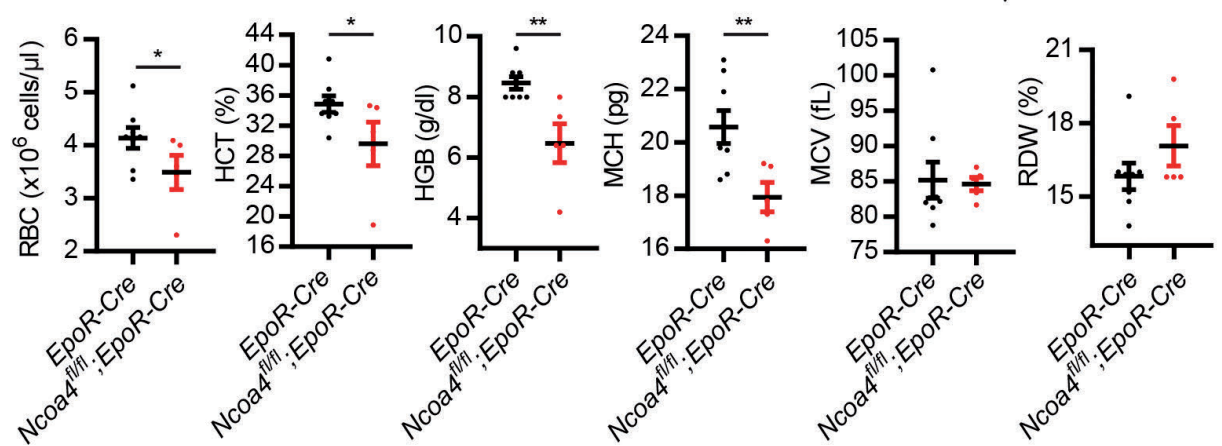

Figure 4. Ncoa $4^{f / f f} ;$;EpoR-Cre mice reveal a cell autonomous role of Ncoa4 in erythropoiesis. A murine model of Ncoa4 deficiency in Ter119+ cells was generated by

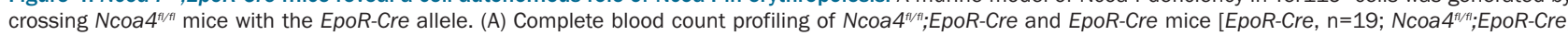
$\mathrm{n}=21$ mice/group, error bars represent the standard error of mean (s.e.m.)]. (B) Erythropoietin levels in serum of EpoR-Cre versus Ncoa4t/fit:EpoR-Cre. (EpoR-Cre, $\mathrm{n}=9$

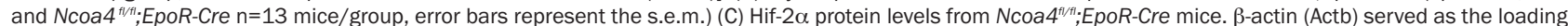
control. (D) Increased Fth1 protein levels in bone marrow from Ncoa $4^{t / f t}$; EpoR-Cre mice. Actb served as a loading control. (E) Increased tissue iron levels in bone marrow from Ncoa4 ${ }^{t / f f} ; E p o R$-Cre mice ( $n=6 /$ group, error bars represent the s.e.m.). (F) Bach1, Hri, Eif2 $\alpha$ and Eif2 $\alpha$-P protein levels in red blood cells of EpoR-Cre and

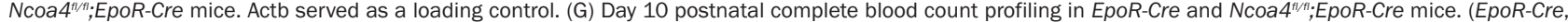
$\mathrm{n}=8$; Ncoa $4^{\mathrm{t} / \mathrm{t} t}$;EpoR-Cre, $\mathrm{n}=5 \mathrm{mice} /$ group, error bars represent the s.e.m.). Statistical comparison was performed using one-tailed Student $t$-test: $* P<0.05$, $\star \star P<0.01$. When not otherwise indicated, data in all panels were subjected to statistical comparison using a two-tailed Student $t$-test: $* P<0.05$, $* \star P<0.01$, $* * * P<0.001$. RBC: red blood cell count; HCT: hematocrit; HGB: hemoglobin; MCH: mean corpuscular hemoglobin; CHr: reticulocyte hemoglobin content; MCV: mean corpuscular volume; RDW: red blood cell distribution width; RETIC: reticulocyte count; PLT: platelet count; WBC: white blood cell count. Epo: erythropoietin; Hif-2 $\alpha$ : heat-inducible factor- $2 \alpha$; Fth1: ferritin heavy chain 1. 
A
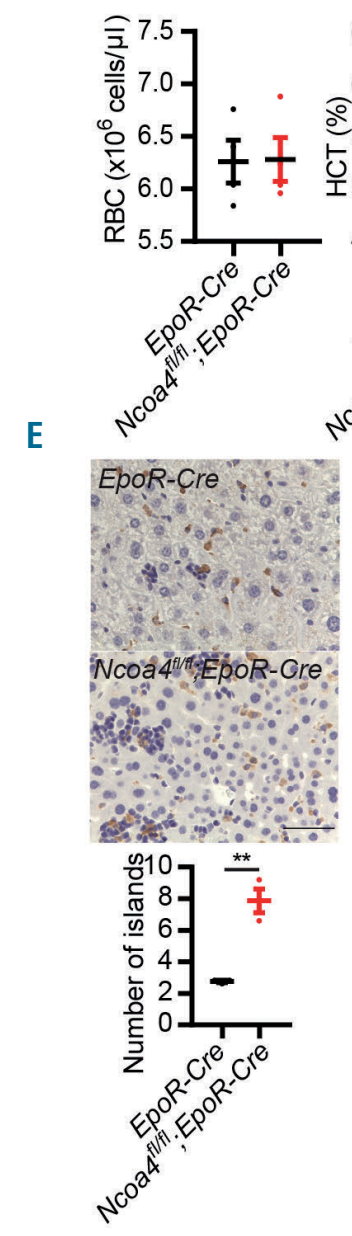

B

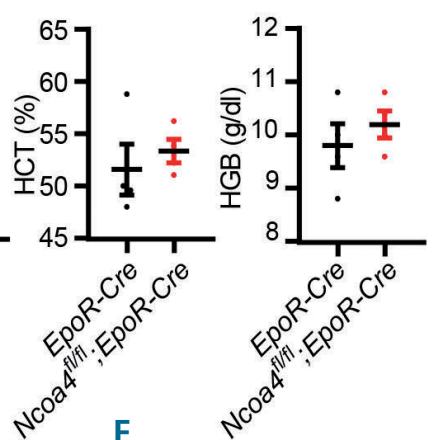

$\mathrm{F}$
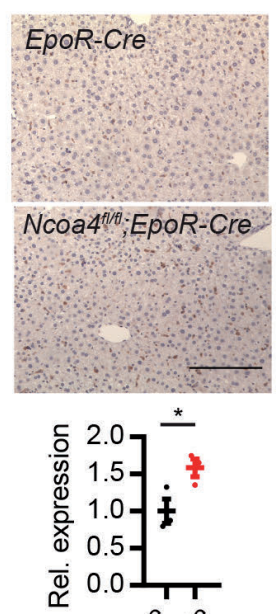

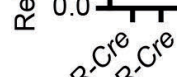

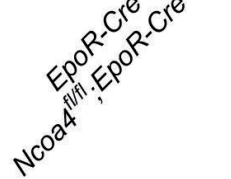

C
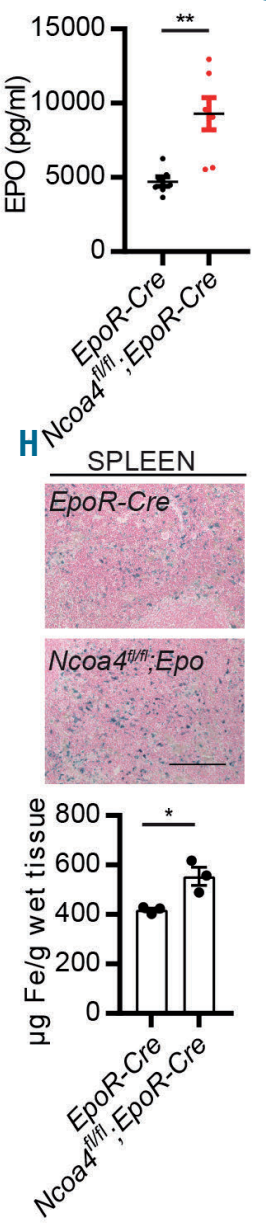

EpoR-Cre Ncoa4 $4_{\text {Epofit; }}^{\text {EpoR-Cre }}$
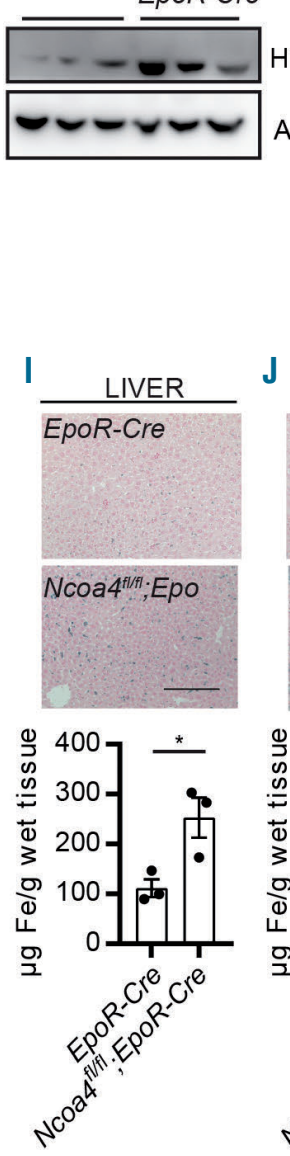

$\mathrm{J}$
Actb
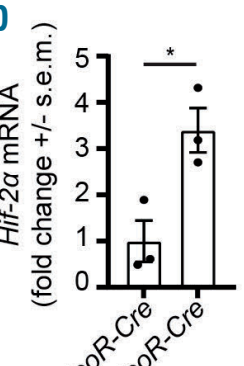

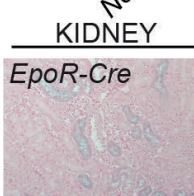

Ncoa $4^{(1 / n)}$;Epo
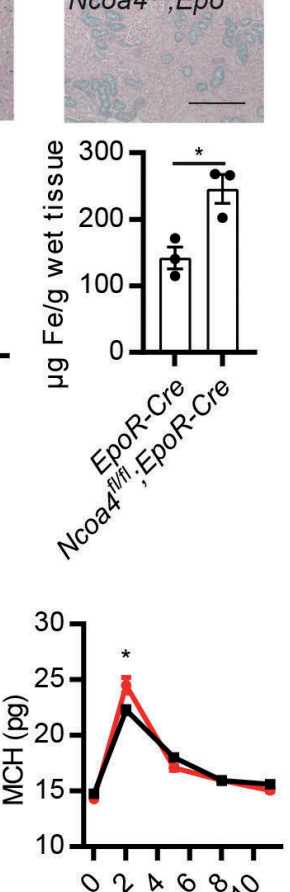

o $2 \times 68,0$
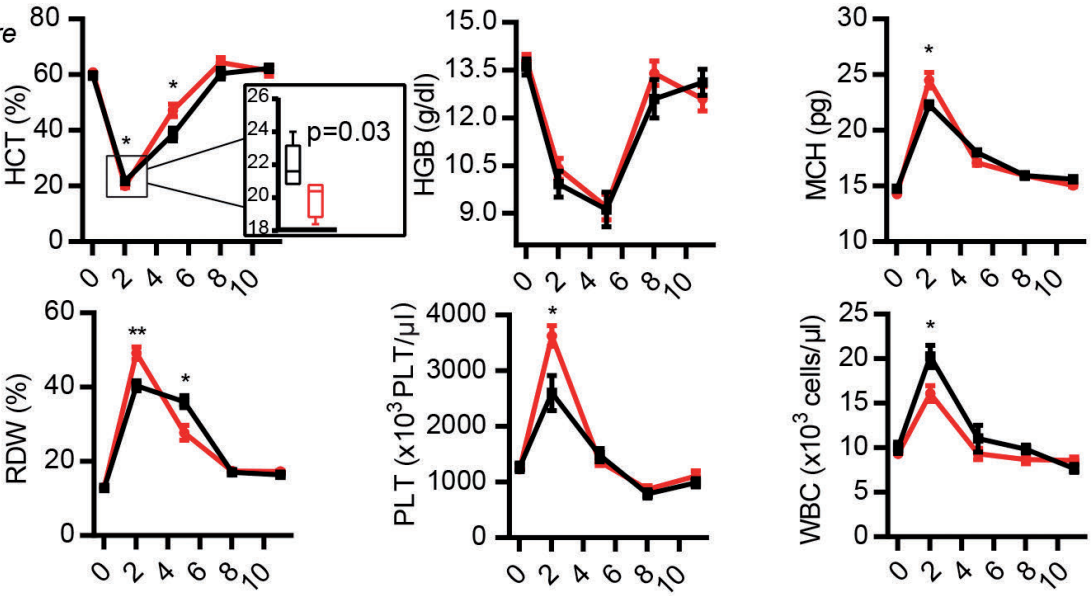

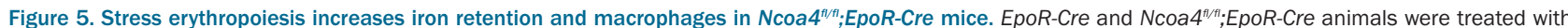
phenylhydrazine $(\mathrm{PHZ})$ to induce red blood cell $(\mathrm{RBC})$ lysis. (A) RBC count, hematocrit, and hemoglobin in Ncoa4 ${ }^{\mathrm{f} / \mathrm{f} / \mathrm{E}}$ :EpoR-Cre and EpoR-Cre mice after PHZ treatment [4 mice/group, error bars represent the standard error of mean (s.e.m.)]. Data are also presented in Online Supplementary Table S7. (B) Erythropoietin levels in

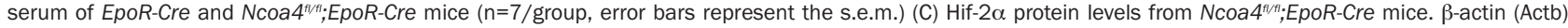

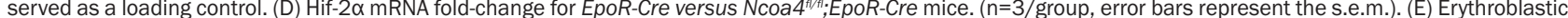


animal (3 mice per group). (Bottom) Quantification of the number of erythroblastic islands (3 mice/group, error bars represent the s.e.m.). (F) Elevated Cd68 staining in liver of EpoR-Cre and Ncoa4 $4^{t / f i t}$;EpoR-Cre mice. Representative field (20x) of 3 mice/group (scale bar=200 $\mu \mathrm{m}$ ) Bottom: Relative Cd68 expression of CD68 EpoRCre and Ncoa4 ${ }^{t / f t}$;EpoR-Cre mice ( $\mathrm{n}=3$ /group, error bars represent the s.e.m.). (G) Serial complete blood count profiling of PHZ-administered EpoR-Cre and

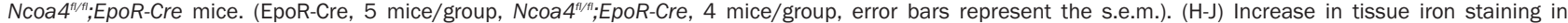
Ncoa $4^{\mathrm{fl} / \mathrm{f}}$;EpoR-Cre mice as determined by Prussian blue staining of spleen (H), liver (I) and kidney (J) (representative fields of 3 mice/group, $10 x$, scale bar=200 $\mu$ m). (Bottom) Tissue iron levels in spleen, liver, and kidney (3 mice/group, error bars represent the s.e.m.). For all panels, statistical comparison was performed using a two-tailed Student $t$-test: $* P<0.05, * * P<0.01, * \star * P<0.001$, except for panel G (hematocrit, day 2), where a one-tailed Student $t$-test was performed: $* P<0.05$. RBC: red blood cell count; HCT: hematocrit; HGB: hemoglobin; EPO: erythropoietin; Hif-2 $\alpha$ : heat-inducible factor-2A; MCH: mean corpuscular hemoglobin; $\mathrm{CHr}$ : reticulocyte hemoglobin content; MCV: mean corpuscular volume; RDW: red blood cell distribution width; PLT: platelet count; WBC: white blood cell count. 
(Online Supplementary Figure S4A) and Epo levels (Online Supplementary Figure S4B) normalized suggesting complete recovery. No differences were observed in the differentiation profile and number of precursors in bone marrow (Online Supplementary Figure S4C).

Consistent with the UBC-Cre/ERT2 model, we observed a lower hematocrit nadir in the Ncoa $4^{f p r}$;EpoR-Cre model in comparison to that of the control. Consistent with previous reports, ${ }^{34}$ hematocrit levels recovered 3 days after nadir, which is in contrast with the systemic KO model in which the hematocrit level decreased even further in the same period of time, coincident with a delay in reticulocytosis. These data suggest that reticulocytosis is not impaired in Ncoa ${ }^{A f r} ;$;EpoR-Cre mice, a model in which tissue Ncoa4 expression and systemic iron metabolism are intact. On the other hand, in the Ncoa ${ }^{f(n)}$; EpoR-Cre model Prussian blue staining (Figure 5H-J, top) and tissue iron levels (Figure $5 \mathrm{H}-\mathrm{J}$, bottom) were increased after PHZ induction. Non-heme iron in RBC from Ncoa $4^{q p /}$; EpoR-Cre animals was not elevated (Online Supplementary Figure $S 4 D$ ), suggesting that elevated tissue iron is not a consequence of elevated iron in RBC but more likely due to increased hemolysis. Overall these data suggest that RBC from Ncoa ${ }^{t r}$; EPoR-Cre mice are more sensitive to PHZinduced lysis.

\section{NCOA4 depletion impairs hemoglobinization and redox balance in $\mathrm{K} 562$ cells}

In order to develop a deeper understanding of the cell autonomous role of NCOA4, we depleted NCOA4 in a K562 cellular model of erythropoiesis and analyzed the proteomic changes under basal and differentiation conditions. Using mass spectrometry-based quantitative proteomics, we identified and quantified 7,869 proteins (Online Supplementary Table S8). NCOA4 was depleted in both basal and hemin-differentiated conditions (Online Supplementary Table S9). Hemoglobin subunits (HBD, HBE1, HBZ) were significantly decreased in NCOA4depleted cells (Figure 6A, Online Supplementary Table S9) as were the associated GSEA pathways (hemoglobin complex and AHSP pathway: hemoglobin chaperone system) (Figure 6B, Online Supplementary Figure S5A). Several heme synthesis pathway proteins were decreased under basal conditions, including FECH and UROS (Figure 6A, Online Supplementary Table S9). Likewise, the heme metabolism GSEA pathway was downregulated (Figure 6B, Online Supplementary Figure S5A). These results are consistent with a phenotypic defect in hemoglobinization of NCOA4-depleted K562 cells and in vivo in erythroid cells and aligns with a defect in iron mobilization to mitochondria to support heme synthesis.,

We next used erythroid differentiation stage-specific proteomic maps to analyze the effects of NCOA4 depletion during differentiation. ${ }^{23}$ Control cells treated with hemin (shGFP+HM vs. shGFP) showed the strongest correlation with advanced stages of erythropoiesis whereas NCOA4-depleted cells (shNCOA4 vs. shGFP) were associated with early-stage progenitor proteomes (Figure 6C, Online Supplementary Table S10). In comparison to basal conditions, NCOA4-depleted cells treated with hemin (shNCOA4+HM vs. shGFP+HM) were closer to the laterstage proteomes showing that there was some ability of NCOA4-depleted cells to differentiate (Figure 6C, Online Supplementary Figure S5B, Online Supplementary Table S10).

We further analyzed proteomic changes for potential molecular explanations of the increased oxidant sensitivity of Ncoa4-deficient erythrocytes. Catalase, important for mitigating oxidative stress in erythroid cells, was significantly decreased in NCOA4-depleted cells (Figure 6A, Online Supplementary Table S9). ${ }^{40,41}$ The decrease in catalase is likely due to reduced ferritinophagy which decreases bioavailable iron and thereby decreases the basal accumulation of reactive oxygen species by the Fenton reaction of the Haber-Weiss cycle. ${ }^{42,43}$ We next determined basal and induced levels of reactive oxygen species in RBC from Ncoa $4^{p / p}$;EpoR-Cre and EpoR-Cre mice. While a decrease in basal levels of catalase could portend increased sensitivity to redox stress, there was no significant increase in baseline or induced reactive oxygen species levels. On the contrary, consistent with prior results in NCOA4-depleted cancer cell lines, RBC from Ncoa $4^{f / 1} ; E$;EOR-Cre mice appeared resistant to direct challenge by reactive oxygen species (Figure 6D). However, there were no differences in reactive oxygen species levels (Figure $6 \mathrm{E}$ ) in response to ex vivo PHZ challenge. Further investigation is warranted to determine the cause of the increased sensitivity of Ncoa4depleted RBC to PHZ

\section{Discussion}

Our data support a model of both cell autonomous and non-autonomous roles for Ncoa4 in erythropoiesis and establish the importance of Ncoa4 in regulating basal systemic iron homeostasis. Prior data from cell culture model systems revealed an important role for constitutive activity of Ncoa4-mediated ferritinophagy in supporting basal intracellular iron homeostasis. Using a tamoxifeninducible murine model of Ncoa4 depletion, we now demonstrate that there is a similar constitutive flux through the Ncoa4-mediated ferritinophagy pathway in vivo to support systemic iron homeostasis. We further demonstrate that there appears to be variable flux through this pathway depending on the organ. After acute Ncoa4 ablation, Fth1 accumulates in liver, spleen, and kidney but not in the pancreas or bone marrow and to a varying degree in the brain. This suggests a higher basal flux through the ferritinophagy pathway in specific tissues involved in systemic iron homeostasis. Further exploration of additional tissues and specific cell types under basal and stimulated conditions will be instructive regarding the tissues with the highest basal and stimulated requirements for ferritinophagy for maintaining intracellular iron homeostasis. Furthermore, our work supports additional study of the role of Ncoa4 under pathological conditions in which iron homeostasis is disrupted, such as hemochromatosis and iron deficiency anemia. ${ }^{44}$

Impaired whole body Ncoa4 function induces a hypochromic microcytic anemia consistent with an iron deficiency anemia despite tissue iron overload, highlighting the importance of Ncoa4-mediated ferritinophagy in supporting erythropoiesis. ${ }^{12}$ Our data are the first to show that Ncoa4 has both cell autonomous and nonautonomous roles in erythropoiesis. Constitutive targeted ablation of Ncoa4 in the erythroid compartment leads to a pronounced anemia in postnatal stages and a mild hypochromic microcytic anemia in adult mice. The impaired hemoglobinization of RBC (low mean corpuscular hemoglobin) and reticulocytes (reticulocyte hemoglobin content) shows that Ncoa4-mediated fer- 
ritinophagy is an important step in vivo in the intra-erythrocyte itinerary and utilization of iron for heme synthesis. This aligns with in vitro data showing that ferritin is an important intermediate step in iron destined for heme synthesis. ${ }^{8,9}$ In comparison to the anemia in a constitutive systemic Ncoa4 KO mouse model, the anemia in our erythroid targeted model is less severe, also suggesting a non-cell autonomous role of Ncoa4 in supporting erythropoiesis.

Given the constitutive $\mathrm{KO}$ nature of prior model systems, it was initially unclear whether the observed microcytic anemia in adult Ncoa4-deficient mice was an early or late consequence of Ncoa4 depletion. Gao et al. recently showed that the most severe defect in erythropoiesis is in the immediate postnatal period, highlighting the importance of accounting for temporal variations in the requirement for Ncoa4 function. ${ }^{13}$ Similarly, targeted deletion of Ncoa4 in the erythroid compartment provokes a more significant anemia in postnatal mice, albeit less severe than that of the systemic knockout model. The severity of the phenotype in the postnatal period is likely a consequence of the significant demand for iron mobilization from ferritin in all tissues, in particular from the liver where iron stores are high in neonatal mice, to rapidly expand the erythroid compartment. ${ }^{45}$ In this prior study, Gao et al. proposed a new role for Ncoa4 in mediating a thyroid hor-
A

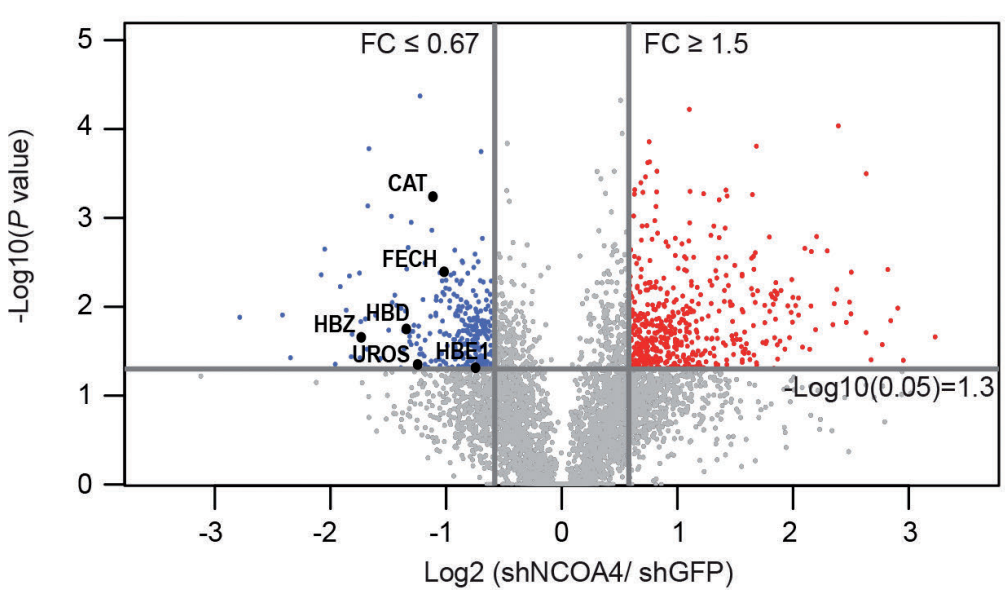

C

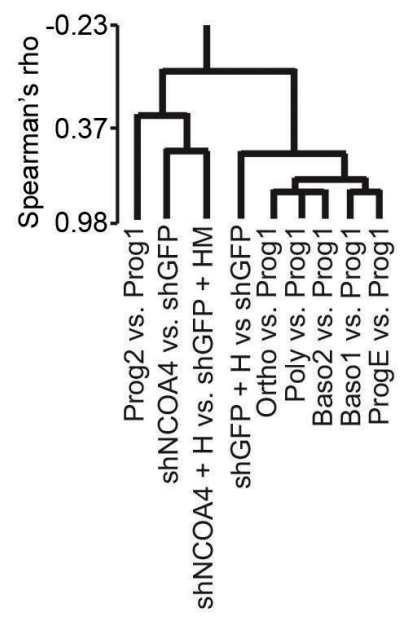

B

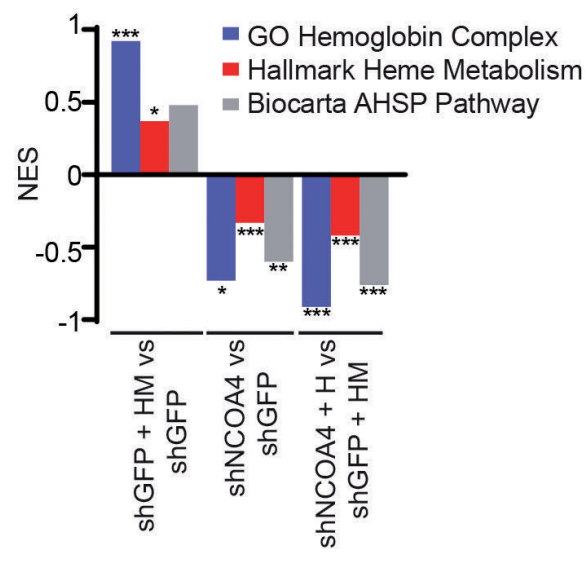

$\mathbf{E}$

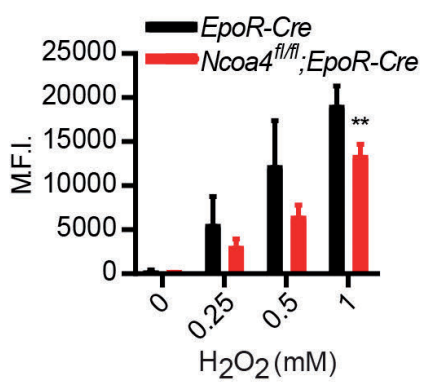

Figure 6. Depletion of NCOA4 impairs erythroid differentiation and heme synthesis in K562 cells. Proteome comparison of K562 cells expressing short hairpin (sh) NCOA4 or shGFP (control) shows significant reduction of proteins required for hemoglobin synthesis and erythroid differentiation. (A) Volcano plot of differentially expressed proteins in shNCOA4- versus shGFP-expressing cells in basal conditions. The cutoff criteria to enter the analysis was an analysis of variance (ANOVA) false discovery rate $<0.05$. Upregulated proteins [fold change (FC) $\geq 1.5$ and post-hoc $P$ value $<0.05$ ] are represented in red and downregulated (FC $\leq 0.67$ and a post-hoc $P$ value $<0.05$ ) are represented in blue. (B) Gene set enrichment analysis (GSEA) results of pathways associated with hemoglobin synthesis: GO Hemoglobin complex (blue), Hallmark Heme metabolism (red) and Biocarta AHSP pathway (gray). (C) The dendrogram shows the relative distance of pre-ranked GSEA results with the nonparametric Spearman rank correlation coefficient rho used as a distance metric (see Methods). Correlation coefficients of differentially expressed proteomes: shGFP + hemin (HM) versus shGFP, shNCOA4 versus shGFP and shNCOA4 + HM versus shGFP + HM were compared to proteomic data of seven erythropoietic stages: burstforming unit-erythroid (Prog1); colony-forming unit-erythroid (Prog2), proerythroblast (ProE), basophilic erythroblast (Baso), polychromatic erythroblast (Poly) and orthochromatic erythroblast (Ortho). (D-E) Mean fluorescence intensity (M.F.I.) of DCFH-DA-stained red blood cells from EpoR-Cre and Ncoa4t/fif:EpoR-Cre mice was measured after no treatment, treatment with $\mathrm{H}_{2} \mathrm{O}_{2}(D)$, or treatment with phenylhydrazine $(E)$. Statistical comparison was performed using a two-tailed Student $t$-test: $* P<0.05, * * P<0.01$. NES: normalized enrichment score; $\mathrm{H}_{2} \mathrm{O}_{2}$ : hydrogen peroxide; PHZ: phenylhydrazine. 
mone-dependent transcriptional program that supports erythropoiesis. While our results do not exclude this possibility, it is clear that the erythroid-specific function of Ncoa4 does not account for the entirety of the anemia effect in systemic Ncoa4 KO mice. These results will have to be reconciled with the clear role of Ncoa4 in mediating ferritinophagy ${ }^{7,8}$ as well as reports that $\mathrm{Ncoa} 4$ participates in DNA replication origin activation. ${ }^{46}$ Contrasting these results from the postnatal stage with those from adult mice, Ncoa4-deficient mice recover from the profound postnatal anemia either because they are no longer dependent on cell autonomous or non-autonomous Ncoa4 function or because compensatory mechanisms replace Ncoa4 function. For the first time, we are now able to temporally control Ncoa4 ablation. Our results show that Ncoa4 is important in the adult mouse given that an acute ablation of Ncoa4 leads to anemia. While we have not determined the cause of the acute anemia it is interesting to speculate based on results from Gao et al. that show a block in terminal differentiation of primary human erythroblasts upon acute NCOA4 knockdown, which may render a subset of newly synthesized reticulocytes particularly sensitive to turnover.

The role of Ncoa4 in erythroid differentiation has been studied intensively with mixed results. Our results with targeted constitutive ablation of Ncoa4 in the erythroid compartment showed no appreciable defect in differentiation of erythrocyte precursors derived from an adult mouse. Likewise, we saw no effect on erythroid differentiation after acute systemic Ncoa4 depletion. Our results could suggest a number of possibilities with respect to the role of Ncoa4 in erythroid differentiation: (i) erythroid intrinsic Ncoa4 function may not be important for in vivo differentiation; (ii) erythroid intrinsic Ncoa4 function in differentiation may be dispensable and compensated for by other intrinsic or extrinsic mechanisms; and/or (iii) erythroid differentiation is dependent on $\mathrm{Ncoa} 4$ in postnatal mice but not in adult mice. Erythroid compartment-specific Ncoa4 rescue in the setting of a Ncoa4 null mouse may answer the question of extrinsic effects on differentiation.

Overall, we have shown that Ncoa4 is important for basal and stimulated erythropoiesis by regulating both systemic and RBC iron metabolism. Loss of Ncoa4 triggers significant compensatory mechanisms to mitigate baseline and induced anemias. In response to Ncoa4 depletion, the Hif-2 $\alpha$-Epo axis is upregulated to compensate for anemia. We note that with a concurrent iron deficiency, activation of Irp1 may repress Hif- $2 \alpha$ translation; however, the net result in our system is induction of Hif- $2 \alpha$ protein expression. Activation of the Hif- $2 \alpha$-Epo pathway mediates a compensatory increase in Fpn expression, which increases iron availability from tissues and iron import from the diet. Furthermore, both the Epo system and extramedullary erythropoiesis are required for recovery from chemicallyinduced anemias in Ncoa4-null mice. As Ncoa4-depleted RBC maintain their ability to synthesize heme and hemoglobin, there must exist additional baseline or compensatory pathways to ensure continued iron delivery to the mitochondria. Our erythroid compartment-specific mouse model shows a cell autonomous role of Ncoa4 but also highlights the non-autonomous role of Ncoa4 in maintaining erythropoiesis. Future work will be directed at determining the non-autonomous contribution of Ncoa4 to erythropoiesis, with the liver (hepatocytes) and macrophages being two likely cell types given their established roles in systemic iron homeostasis.

\section{Acknowledgments}

We thank Roderick Bronson in the Rodent Histopathology Core at the Dana-Farber/Harvard Cancer Center in Boston, MA for tissue processing and histopathological interpretation services. We thank Steven Gygi for use of CORE for mass spectrometry data analysis. This work was supported by a Burroughs Wellcome Fund Career Award for Medical Scientists, Brigham and Women's Hospital MFCD Award, Sidney Kimmel Foundation Kimmel Scholar Program, and Dana-Farber Cancer Institute Claudia Adams Barr Program for Innovative Cancer Research Award to JDM, and NIH grant R01GM095567 to $A C K$ and JWH WF and BM are supported by the First TEAM of the Foundation for Polish Science. Dana-Farber/Harvard Cancer Center is supported in part by a NCI Cancer Center Support Grant \# NIH 5 P3O CA06516.

\section{References}

1. Muckenthaler MU, Rivella S, Hentze MW, Galy B. A red carpet for iron metabolism. Cell. 2017;168(3):344-361.

2. Pantopoulos K, Porwal SK, Tartakoff A, Devireddy L. Mechanisms of mammalian iron homeostasis. Biochemistry. 2012;51 (29):5705-5724.

3. Camaschella C. New insights into iron deficiency and iron deficiency anemia. Blood Rev. 2017;31(4):225-233.

4. Weis S, Carlos AR, Moita MR, et al. Metabolic adaptation establishes disease tolerance to sepsis. Cell. 2017;169(7):12631275

5. Theil E. Ferritin: the protein nanocage and iron biomineral in health and in disease. Inorg Chem. 2013;52(21):12223-12233.

6. Dowdle WE, Nyfeler B, Nagel J, et al. Selective VPS34 inhibitor blocks autophagy and uncovers a role for NCOA4 in ferritin degradation and iron homeostasis in vivo. Nat Cell Biol. 2014;16(11):1069-1079.
7. Mancias JD, Wang X, Gygi SP, Harper JW, Kimmelman AC. Quantitative proteomics identifies NCOA4 as the cargo receptor mediating ferritinophagy. Nature. 2014;509 (7498):105-109.

8. Mancias JD, Pontano Vaites L, Nissim S, et al. Ferritinophagy via NCOA4 is required for erythropoiesis and is regulated by iron dependent HERC2-mediated proteolysis. Elife. 2015;4.

9. Ryu MS, Zhang D, Protchenko O, Shakoury-Elizeh M, Philpott CC. PCBP1 and NCOA4 regulate erythroid iron storage and heme biosynthesis. J Clin Invest. 2017;127(5):1786-1797

10. Weber GJ, Choe SE, Dooley KA, PaffettLugassy NN, Zhou Y, Zon LI. Mutant-specific gene programs in the zebrafish. Blood. 2005;106(2):521-530.

11. Nilsson R, Schultz IJ, Pierce EL, et al. Discovery of genes essential for heme biosynthesis through large-scale gene expression analysis. Cell Metab. 2009;10(2): 119-130.
12. Bellelli R, Federico G, Matte A, et al. NCOA4 deficiency impairs systemic iron homeostasis. Cell Rep. 2016;14(3):411-421.

13. Gao X, Lee H, Li W, Jeffrey R, Barrasa MI $\mathrm{Ma} \mathrm{Q}$. Thyroid hormone receptor beta and NCOA4 regulate terminal erythrocyte differentiation. Proc Natl Acad Sci U S A. 2017;114(38):10107-10112

14. Heinrich AC, Pelanda R, Klingmüller U. A mouse model for visualization and conditional mutations in the erythroid lineage. Blood. 2004;104(3):659-666.

15. Cook JD. Methods in Hematology. 1980.

16. Liu J, Zhang J, Ginzburg Y, et al. Quantitative analysis of murine terminal erythroid differentiation in vivo: novel method to study normal and disordered erythropoiesis. Blood. 2015;121(8):43-50.

17. Chen K, Liu J, Heck S, Chasis JA, An X, Mohandas N. Resolving the distinct stages in erythroid differentiation based on dynamic changes in membrane protein expression during erythropoiesis. Proc Natl Acad Sci U S A. 2009;106(41):17413-17418. 
18. Yang A, Rajeshkumar N V, Wang X, et al. Autophagy is critical for pancreatic tumor growth and progression in tumors with p53 alterations. Cancer Discov. 2014;4(8):1-9.

19. Biancur DE, Paulo JA, Małachowska B, et al. Compensatory metabolic networks in pancreatic cancers upon perturbation of glutamine metabolism. Nat Commun. 2017;8: 15965.

20. Paulo J, Mancias J, Gygi S. Proteome-wide protein expression profiling across five pancreatic cell lines. Pancreas. 2017;46(5):690698.

21. McAlister GC, Nusinow DP, Jedrychowski MP, et al. MultiNotch MS3 enables accurate, sensitive, and multiplexed detection of differential expression across cancer cell line proteomes. Anal Chem. 2014;86(14):7150-7158.

22. Subramanian P, Tamayo P, Mootha, VK, et al. Gene set enrichment analysis: a knowledge-based approach for interpreting genome-wide expression profiles. Proc Natl Acad Sci U S A. 2005;102(43):15545-15550.

23. Gautier EF, Ducamp S, Leduc M, et al. Comprehensive proteomic analysis of human erythropoiesis. Cell Rep. 2016;16(5): 1470-1484.

24. An X, Schulz VP, Li J, et al. Global transcriptome analyses of human and murine terminal erythroid differentiation. Blood. 2014;123(22):3466-3477.

25. Beguin Y. Erythropoietin and platelet production. Haematologica. 1999;84(6):541547.

26. Stohlawetz P, Dzirlo L, Hergovich N, et al. Effects of erythropoietin on platelet reactivity and thrombopoiesis in humans. Blood. 2000;95(9):2983-2989

27. Ghosh MC, Zhang DL, Ollivierre $\mathrm{H}$, Eckhaus MA, Rouault TA. Translational repression of HIF2 $\alpha$ expression in mice with Chuvash polycythemia reverses poly- cythemia. J Clin Invest. 2018;128(4):13171325 .

28. Kobayashi M, Kato H, Hada H, et al. Ironheme-Bach1 axis is involved in erythroblast adaptation to iron deficiency. Haematologica. 2017;102(3):454-465.

29. Zhang S, Macias-Garcia A, Velazquez Paltrinieri E, Kaufman RJ, Chen J-J. HRI coordinates translation by eIF $2 \alpha \mathrm{P}$ and mTORC1 to mitigate ineffective erythropoiesis in mice during iron deficiency. Blood. 2018;131(4):450-461.

30. Korolnek T, Hamza I. Macrophages and iron trafficking at the birth and death of red cells. Blood. 2016;125(19):2893-2898.

31. Chow A, Huggins M, Ahmed J, et al CD169+ macrophages provide a niche promoting erythropoiesis under homeostasis and stress. Nat Med. 2013;19(4):429-436.

32. Theurl I, Hilgendorf I, Nairz M, et al. Ondemand erythrocyte disposal and iron recycling requires transient macrophages in the liver. Nat Med. 2016;22(8)945-951.

33. Paulson R, Shi L, Wu D-C. Stress erythropoiesis: new signals and new stress progenitor cells. Curr Opin Hematol. 2011;18(3): 139-145.

34. Frazer D, Inglis $\mathrm{H}$, Wilkins $\mathrm{S}$, et al. Delayed hepcidin response explains the lag period in iron absorption following a stimulus to increase erythropoiesis. Gut. 2004;53(10): 1509-1515.

35. Zhang D, Meyron-Holtz EG, Rouault TA. Renal iron metabolism: transferrin iron delivery and the role of iron regulatory proteins. J Am Soc Nephrol. 2007;18(2):401-406.

36. Oian $\mathrm{Q}$, Nath K, Wu Y, Daoud T, Sethi S. Hemolysis and acute kidney failure. Am J Kidney Dis. 2010;56(4):780-784.

37. Ramos P, Casu C, Gardenghi S, et al. Macrophages support pathological erythropoiesis in polycythemia vera and $\beta$-tha- lassemia. Nat Med. 2013;19(4):437-445

38. Sonoda Y, Sasaki K. Hepatic extramedullary hematopoiesis and macrophages in the adult mouse: histometrical and immunohistochemical studies. Cells Tissues Organs. 2012;196(6):555-564.

39. Klei $T$, Meinderts $S$, van den Berg $T$, van Bruggen R. From the cradle to the grave: the role of macrophages in erythropoiesis and erythrophagocytosis. Front Immunol. 2017;8:73.

40. Mueller S, Riedel H, Stremmel V. Direct evidence for catalase as the predominant $\mathrm{H} 2 \mathrm{O} 2$ -removing enzyme in human erythrocytes. Blood. 1997;90(12):4973-4978.

41. Johnson $R$, Ho $Y, Y u$ D, Kuypers $F$ Ravindranath Y, Goyette G. The effect of disruption of genes for peroxiredoxin-2, glutathione peroxidase- 1 and catalase on erythrocyte oxidative metabolism. Free Radic Biol Med. 2010;48(4):519.

42. Fenton $\mathrm{HJH}$. Oxidation of tartaric acid in presence of iron. J Chem Soc Trans. 1894;65:899-910.

43. Sadrzadeh SM, Graf E, Panter SS, Hallaway PE, Eaton JW. Hemoglobin. A biologic Fenton reagent. J Biol Chem. 1984;259(23): 14354-14356.

44. Doulatov S, Vo L, Macari E, et al. Drug discovery for Diamond-Blackfan anemia using reprogrammed hematopoietic progenitors. Sci Transl Med. 2017;9(376).

45. Chakrabarti M, Cockrell A, Park J, McCormick S, Lindahl L, Lindahl P. Speciation of iron mouse liver during development, iron deficiency, IRP2 deletion and inflammatory hepatitis. Metallomics. 2015;7(1):88-96

46. Bellelli R, Castellone MD, Guida T, et al NCOA4 transcriptional coactivator inhibits activation of DNA replication origins. Mol Cell. 2014;55(1):123-137. 